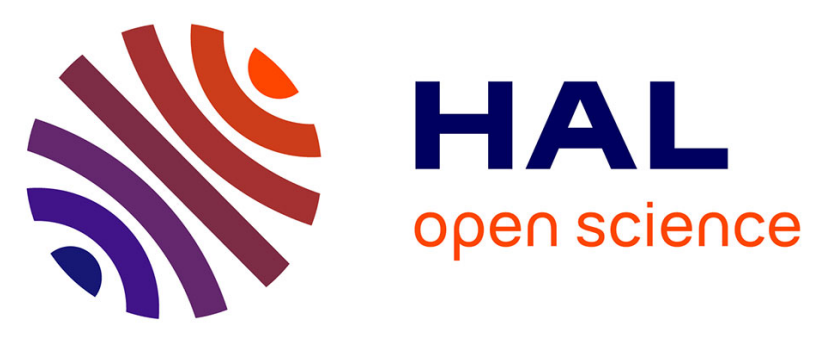

\title{
Conditions for the Palladium-Catalysed Direct 2-Arylation of 3-Bromobenzo[b]thiophene Tolerant of the Benzothienyl Carbon-Bromine Bond
}

Imen Smari, Hamed Ben Ammar, Bechir Ben Hassine, Jean-François Soulé, Henri Doucet

\section{To cite this version:}

Imen Smari, Hamed Ben Ammar, Bechir Ben Hassine, Jean-François Soulé, Henri Doucet. Conditions for the Palladium-Catalysed Direct 2-Arylation of 3-Bromobenzo[b]thiophene Tolerant of the Benzothienyl Carbon-Bromine Bond. Synthesis: Journal of Synthetic Organic Chemistry, 2015, 47 (21), pp.3354-3362. 10.1055/s-0034-1378733 . hal-01188188

HAL Id: hal-01188188

https://hal-univ-rennes1.archives-ouvertes.fr/hal-01188188

Submitted on 16 Dec 2015

HAL is a multi-disciplinary open access archive for the deposit and dissemination of scientific research documents, whether they are published or not. The documents may come from teaching and research institutions in France or abroad, or from public or private research centers.
L'archive ouverte pluridisciplinaire HAL, est destinée au dépôt et à la diffusion de documents scientifiques de niveau recherche, publiés ou non, émanant des établissements d'enseignement et de recherche français ou étrangers, des laboratoires publics ou privés. 


\title{
Graphical Abstract
}

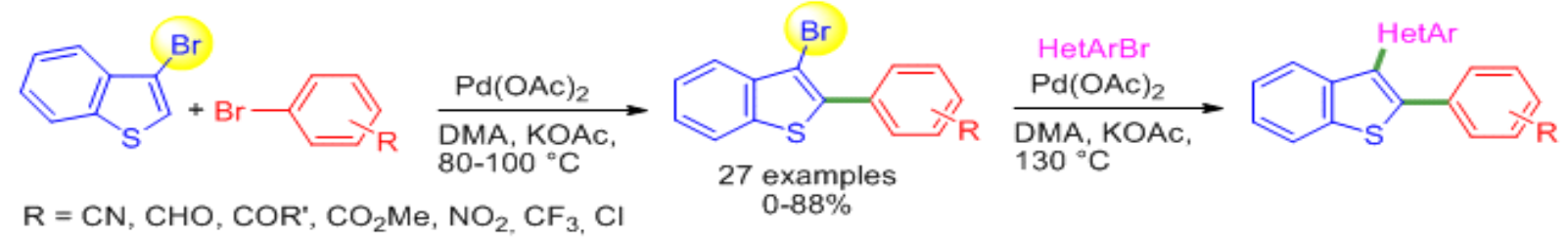

\section{Conditions for Palladium-Catalysed Direct 2-Arylation of 3- Bromobenzothiophene Derivatives Tolerant to the Benzothienyl C-Br bond}

\author{
Imen Smari, ${ }^{\mathrm{a}, \mathrm{b}}$ Hamed Ben Ammar, ${ }^{\mathrm{b}^{*}}$ Bechir Ben Hassine, ${ }^{\mathrm{b}}$ Jean-François Soulé, ${ }^{\mathrm{a}}$ and Henri Doucet ${ }^{\mathrm{a}^{*}}$ \\ ${ }^{a}$ Institut des Sciences Chimiques de Rennes, UMR 6226 CNRS-Université de Rennes, "Organométalliques: Matériaux et \\ Catalyse", Campus de Beaulieu, 35042 Rennes, France. \\ ${ }^{b}$ Laboratoire de Synthèse Asymétrique et catalyse homogène, (UR 11ES56) Université de Monastir, Faculté des Sciences de Monastir, \\ avenue de l'environnement, Monastir 5000, Tunisie. Fax: (+216) 73500 278; Tel: (+216) 73500275.
}

Fax: 33 (2) 23236939

E-mail: henri.doucet@univ-rennes1.fr

Received: The date will be inserted once the manuscript is accepted.

\begin{abstract}
Phosphine-free $\mathrm{Pd}(\mathrm{OAc})_{2}$ catalyst was found to promote the direct 2-arylation of 3-bromobenzothiophene without cleavage of the benzothienyl $\mathrm{C}-\mathrm{Br}$ bond, allowing the synthesis in only one step of 2-aryl-3-bromobenzothiophenes. The best results were generally obtained using a low loading of the palladium catalyst $(0.5 \mathrm{~mol} \%)$, quite low reaction temperatures $\left(80-120^{\circ} \mathrm{C}\right)$ and short reaction times $(0.5-2 \mathrm{~h})$. The reaction proceeds with electron-deficient para- meta- and ortho-substituted aryl bromides and also heteroaryl bromides. The benzothienyl $\mathrm{C}-\mathrm{Br}$ bond has been profitably employed for further palladium-catalysed functionalizations. This strategy allows straightforward synthesis of 2,3-di(hetero)arylated benzothiophenes with two different (hetero)aryl units via sequential catalytic arylations.
\end{abstract}

Key words: Aryl halides, Catalysis, $\mathrm{C}-\mathrm{H}$ activation, Benzothiophenes, Palladium. 


\section{Introduction}

The arylation of heteroaromatics such as benzothiophenes for the access to 2-arylbenzothiophene derivatives is an important research field in organic synthesis due to the biological and physical properties of such compounds. For example, Raloxifene is used in the prevention of osteoporosis and breast cancer, and Arzoxifene also exhibits antiosteoporosis properties (Fig. 1). ${ }^{1}$

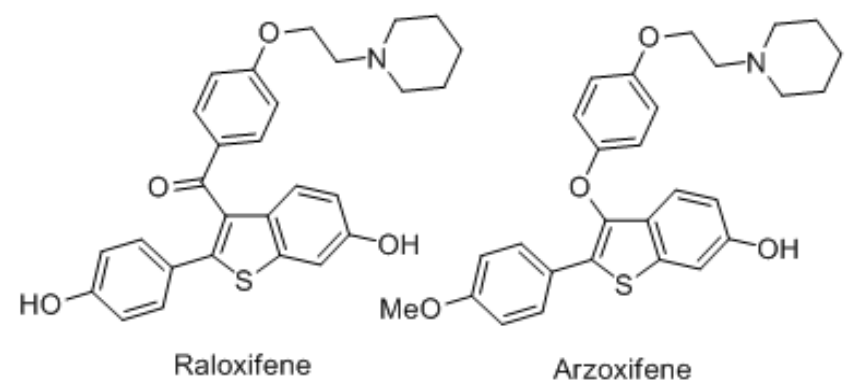

Figure 1 Examples of bioactive 2-arylbenzothiophene derivatives

In 1985-1992, Ohta et al. reported the arylation of several heteroarenes such as thiophenes, furans or thiazoles with aryl halides, via a $\mathrm{C}-\mathrm{H}$ bond activation, in moderate to good yields using $\mathrm{Pd}\left(\mathrm{PPh}_{3}\right)_{4}$ as the catalyst. ${ }^{2 \mathrm{a}, 2 \mathrm{~b}}$ Since these results, the palladium-catalysed direct arylation of heteroaryl derivatives with aryl halides or triflates has proved to be a very effective method for a simpler and "greener" access to arylated heterocycles, due to the reduced number of steps, reduced amount of waste, and to the wider diversity of available compounds. ${ }^{2,3}$ Presently, one of the major drawbacks of this reaction is the functional group tolerance. For example, the use of 3-bromobenzothiophenes for direct C2-arylations, without cleavage of the $\mathrm{C}$-Br bond, would be very useful as it would give a simple access to a wide variety of 3-substituted 2-arylbenzothiophenes useful for pharmaceutical applications. To our knowledge, except our preliminary result which was limited to one specific substrate, ${ }^{4}$ no example of palladium catalysed direct arylations of 3-bromobenzothiophenes has been described (Fig. 1). So far the 2-aryl-3-bromobenzothiophenes were generally prepared via bromination of 2-arylbenzothiophenes using $\mathrm{Br}_{2} .{ }^{5}$ The other methods to prepare such compounds employ the classical palladium-catalysed cross-coupling procedures ${ }^{6}$ such as Suzuki (Scheme 1 , A), ${ }^{7}$ Stille (Scheme 1, B) ${ }^{8}$ or Negishi (Scheme 1, C) ${ }^{9}$ reactions using 2,3-dihalobenzothiophenes (Scheme 1). The Pdcatalysed 2-arylation of 3-bromobenzothiophene with an aryl iodide in the presence of a strong base has also been reported (Scheme 1, D). ${ }^{10}$ As palladium-catalysed direct arylation of easily available 3-bromobenzothiophene would provide a simpler access to 2-aryl-3-bromobenzothiophenes, we decided to investigate its reactivity for such couplings (Scheme 1, E). 

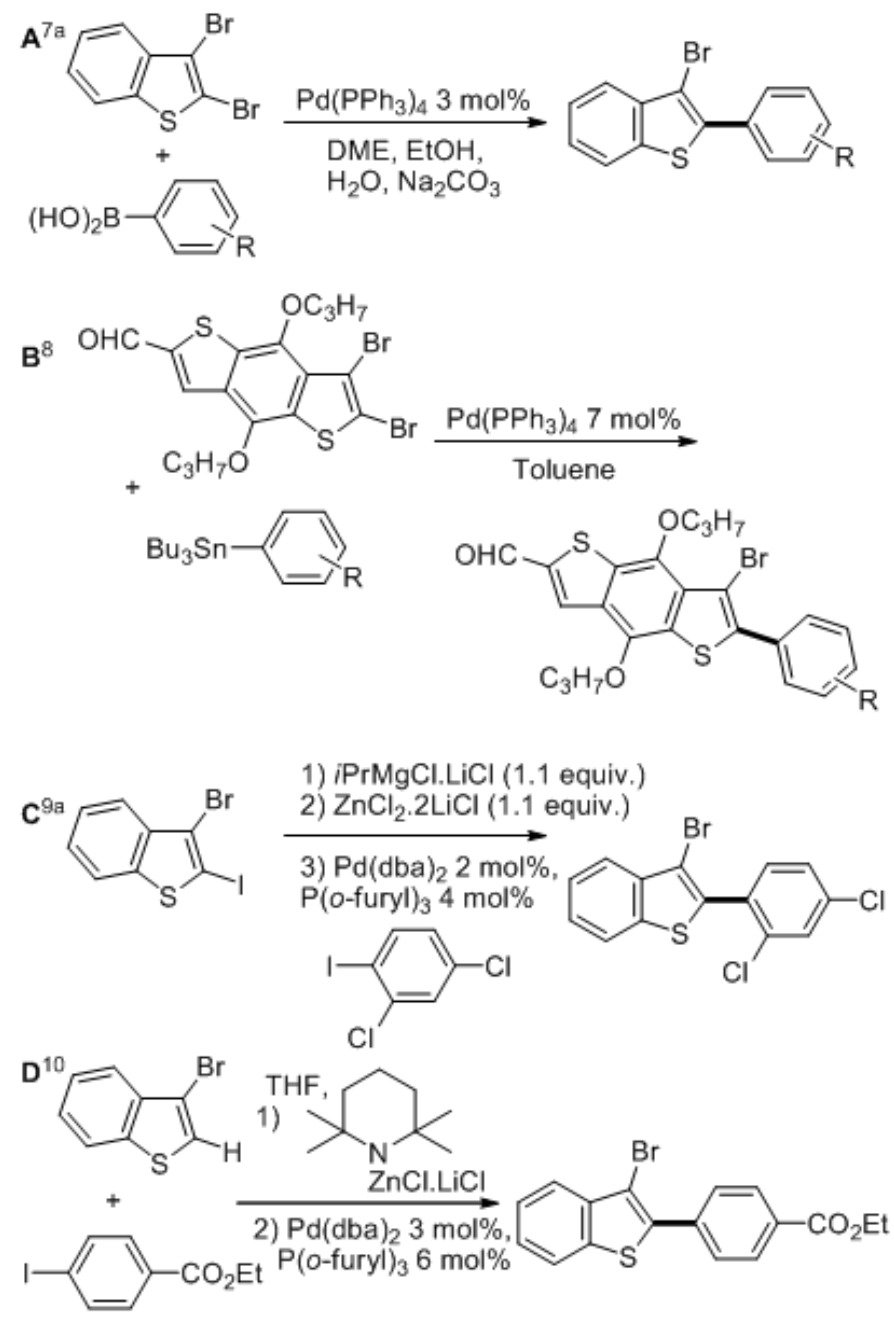

E This work

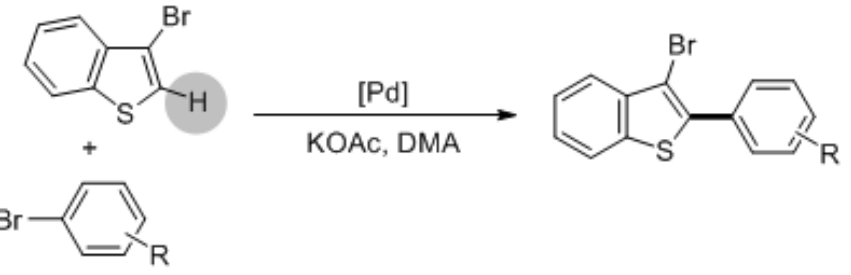

Scheme 1. Reported procedures for Pd-catalysed synthesis of 2-aryl-3-bromobenzothiophenes from 3bromobenzothiophenes 


\section{Results and discussion}

First, we studied the coupling of 3-bromobenzothiophene with 4-bromobenzonitrile using several reaction conditions (Scheme 2, table 1). We have recently reported that the phosphine-free $\mathrm{Pd}(\mathrm{OAc})_{2}$ catalyst reaction conditions allowed the successful coupling of several aryl bromides with simple thiophene derivatives. ${ }^{11}$ Employing $1 \mathrm{~mol} \% \mathrm{Pd}(\mathrm{OAc})_{2}$ as the catalyst and KOAc as the base in DMA (DMA: $N, N$-dimethylacetamide) during $20 \mathrm{~h}$ at $140{ }^{\circ} \mathrm{C}$, we observed the formation of the desired 2-arylated benzothiophene $\mathbf{1}$ in low yield (Scheme 2, Table 1, entry 1). A similar yield was obtained using $\operatorname{PdCl}\left(\mathrm{C}_{3} \mathrm{H}_{5}\right)(\mathrm{dppb})$ catalyst ${ }^{12}$ [dppb: 1,4bis(diphenylphosphino)butane] (Table 1, entry 2). These low yields are probably due to the quite high reactivity of the benzothienyl $\mathrm{C}-\mathrm{Br}$ bond in the presence of a palladium catalyst at this elevated temperature. After the oxidative addition of 3-bromobenzothiophene (or of 1) to palladium, the coupling at $\mathrm{C} 2$ of 3-bromobenzothiophene might produce bis-benzothiophene derivatives as side-products. On the other hand, a reaction time reduced to $2 \mathrm{~h}$ allowed to increase the yield in $\mathbf{1}$ to $36 \%$ with a complete conversion of 4-bromobenzonitrile (Table 1, entry 3 ). Then, several reactions were performed at $120^{\circ} \mathrm{C}$. The best result was obtained using $1 \mathrm{~mol} \% \mathrm{Pd}(\mathrm{OAc})_{2}$ catalyst and $0.5 \mathrm{~h}$ as reaction time (Table 1, entries 4-7). A further decrease of the reaction temperature to $100{ }^{\circ} \mathrm{C}$ and $80{ }^{\circ} \mathrm{C}$ allowed to increase the yield in $\mathbf{1}$ to $72 \%$ and $87 \%$, respectively (Table 1, entries 8 and 9).

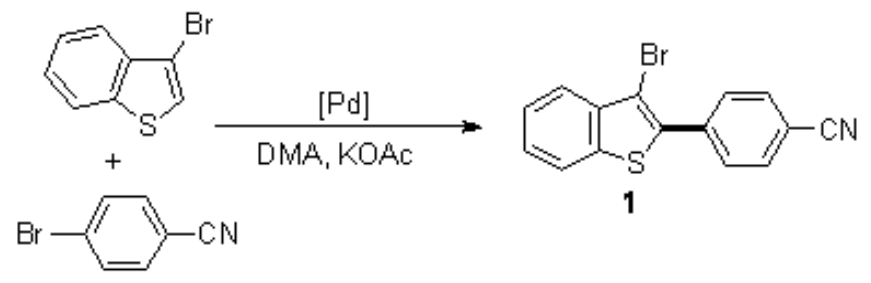

Scheme 2. Influence of the reaction conditions for the coupling of 4-bromobenzonitrile and 3bromobenzothiophene

Table 1. Influence of the reaction conditions for the coupling of 4-bromobenzonitrile and 3-bromobenzothiophene (Scheme 2)

\begin{tabular}{|c|c|c|c|c|c|}
\hline Entry & Catalyst (mol\%) & $\begin{array}{l}\text { Temp. } \\
\left({ }^{\circ} \mathrm{C}\right)\end{array}$ & Time (h) & $\begin{array}{c}\text { Conv. } \\
(\%)\end{array}$ & $\begin{array}{c}\text { Yield in } \\
\mathbf{1}(\%)\end{array}$ \\
\hline 1 & $\mathrm{Pd}(\mathrm{OAc})_{2}(1)$ & 140 & 20 & 100 & 25 \\
\hline 2 & $\begin{array}{c}\operatorname{PdCl}\left(\mathrm{C}_{3} \mathrm{H}_{5}\right) \\
(\mathrm{dppb})(1)\end{array}$ & 140 & 20 & 100 & 23 \\
\hline 3 & $\operatorname{Pd}(\mathrm{OAc})_{2}(1)$ & 140 & 2 & 100 & 36 \\
\hline 4 & $\mathrm{Pd}(\mathrm{OAc})_{2}(1)$ & 120 & 1 & 100 & 57 \\
\hline 5 & $\mathrm{Pd}(\mathrm{OAc})_{2}(1)$ & 120 & 1 & 100 & $48^{\mathrm{a}}$ \\
\hline 6 & $\mathrm{Pd}(\mathrm{OAc})_{2}(0.5)$ & 120 & 1 & 92 & 49 \\
\hline 7 & $\mathrm{Pd}(\mathrm{OAc})_{2}(1)$ & 120 & 0.5 & 100 & 62 \\
\hline 8 & $\mathrm{Pd}(\mathrm{OAc})_{2}(1)$ & 100 & 0.5 & 100 & 72 \\
\hline 9 & $\mathrm{Pd}(\mathrm{OAc})_{2}(1)$ & 80 & 0.5 & 100 & $87(84)$ \\
\hline
\end{tabular}

Conditions: 4-bromobenzonitrile $(1 \mathrm{mmol})$, 3-bromobenzothiophene $(1.5 \mathrm{mmol})$, KOAc $(2 \mathrm{mmol})$, DMA, under argon, GC and NMR yields, yields in parentheses is isolated. a 4-bromobenzonitrile (1.1 mmol), 3bromobenzothiophene (1 mmol). 
Next, we examined the scope and limitations of this reaction using para-, meta- or ortho-substituted aryl bromides and also heteroaryl bromides (Schemes 3-5). 4-Bromobenzaldehyde, 4-bromoacetophenone, 4bromopropiophenone, 4-bromobenzophenone and methyl 4-bromobenzoate gave the desired coupling products 2-6 in $76-88 \%$ yields (Scheme 2, top). 4-Bromonitrobenzene was also a suitable substrate affording 7 in $87 \%$ yield. On the other hand, at $80{ }^{\circ} \mathrm{C}$ the coupling with 4-bromofluorobenzene and 4-bromochlorobenzene only afforded very low yields in 8 and $\mathbf{9}$. The use of higher reaction temperatures $\left(100\right.$ and $\left.130{ }^{\circ} \mathrm{C}\right)$ allowed to slightly improve the yield in 8 to $22 \%$. In the presence of bromobenzene or with the electron-rich 4-bromotoluene, no formation of the desired products $\mathbf{1 0}$ and $\mathbf{1 1}$ was observed even at a higher reaction temperature (Scheme 3, bottom).
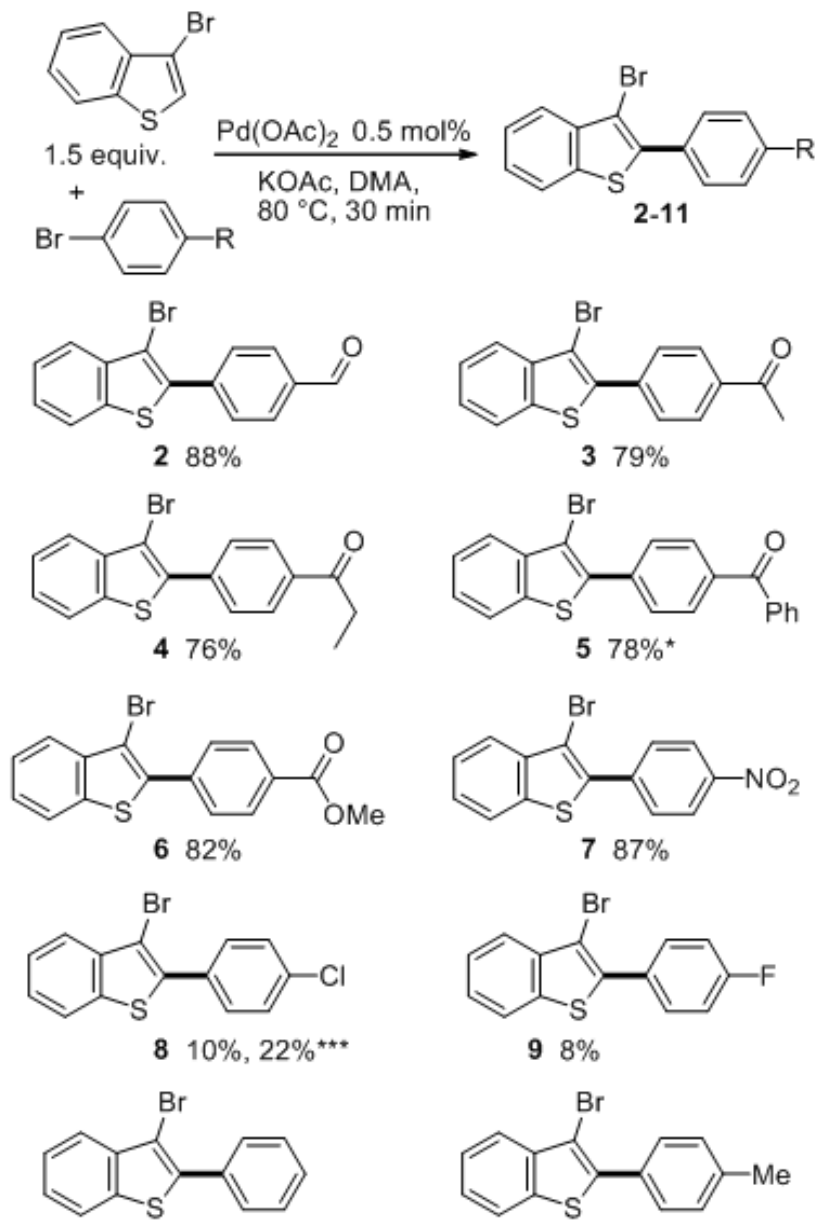

$10 \%$

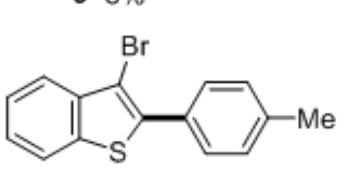

* $120^{\circ} \mathrm{C}, 1 \mathrm{~h}$

$\star \star 100^{\circ} \mathrm{C}, 45 \mathrm{~min}$.

$110 \%, 0 \%$ ***

Scheme 3. Scope of the direct 2-arylation of 3-bromobenzothiophene with para-substituted aryl bromides

Then, the reactivity of a few meta-substituted aryl bromides was investigated (Scheme 4). 3-Bromonitrobenzene, 3-bromobenzonitrile, 3-bromoacetophenone and 3-bromobenzalhedyde also gave the expected 2-arylated benzothiophenes 12-15 in 67-83\% yields. However, with 3-bromonitrobenzene a longer reaction time had to be employed, as after $0.5 \mathrm{~h}$, only a partial conversion this aryl bromide was observed. From the 3,4-disubstituted aryl bromide, 4-bromo-1-nitro-2-(trifluoromethyl)benzene, the product $\mathbf{1 7}$ was also obtained in high yield. Again, fluoro-substituted 3-bromofluorobenzene exhibited a poor reactivity, and $\mathbf{1 8}$ was only produced in $12 \%$ yield. No significant steric effect was observed in the presence of the sterically demanding aryl bromides, 2bromobenzaldehyde and 2-bromobenzonitrile, as $\mathbf{1 9}$ and $\mathbf{2 0}$ were obtained in $80 \%$ and $88 \%$ yields, respectively. Next, we tried to evaluate the difference of reactivity between mono- and di-ortho-substituted aryl bromides, and we were pleased to find that the reaction proceeded efficiently with both 1-bromonaphthalene and 9bromoanthracene to afford $\mathbf{2 1}$ and $\mathbf{2 2}$ in 61 and 64\% yields, respectively. 

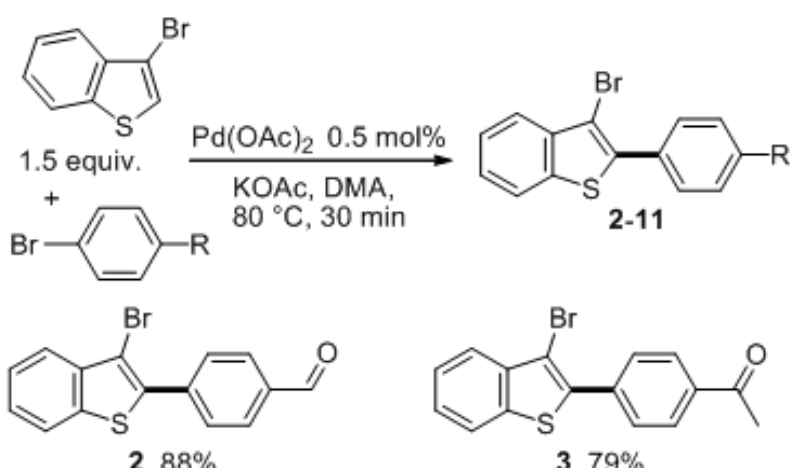

$288 \%$

$379 \%$<smiles>CCC(=O)c1ccc(-c2sc3ccccc3c2Br)cc1</smiles><smiles>O=C(c1ccccc1)c1ccc(-c2sc3ccccc3c2Br)cc1</smiles><smiles>COC(=O)c1ccc(-c2sc3ccccc3c2Br)cc1</smiles><smiles>[Y][R8]c1sc2ccccc2c1-c1ccc([N+](=O)[O-])cc1</smiles><smiles>Clc1ccc(-c2sc3ccccc3c2Br)cc1</smiles><smiles>Fc1ccc(-c2sc3ccccc3c2Br)cc1</smiles>

$98 \%$<smiles>Brc1c(-c2ccccc2)sc2ccccc12</smiles>

$100 \%$<smiles>Cc1ccc(-c2sc3ccccc3c2Br)cc1</smiles>

$110 \%, 0 \%$ ***

* $120^{\circ} \mathrm{C}, 1 \mathrm{~h}$

** $100^{\circ} \mathrm{C}, 45 \mathrm{~min}$

*** $130{ }^{\circ} \mathrm{C}$

Scheme 4. Scope of the direct 2-arylation of 3-bromobenzothiophene with meta- or ortho-substituted aryl bromides

Pyridines are probably the most common heterocyclic motif found in pharmaceutically active compounds. ${ }^{13}$ Therefore, preparative methods of biheteroaryl derivatives containing pyridines remain an essential research topic in organic synthesis. ${ }^{14}$ 3-Bromopyridine and 5-bromopyrimidine were also found to react nicely with 3bromobenzothiophene to give the coupling products $\mathbf{2 3}$ and $\mathbf{2 4}$ in high yields (Scheme 5). 3-Bromoquinoline also affords the desired product $\mathbf{2 5}$ in good yield. Due to a lower reactivity of 4-bromoquinoline, the coupling reaction was performed at $120^{\circ} \mathrm{C}$ during $1 \mathrm{~h}$ to give 26 in $41 \%$ yield. At $100{ }^{\circ} \mathrm{C}$, a lower yield of $20 \%$ was obtained.

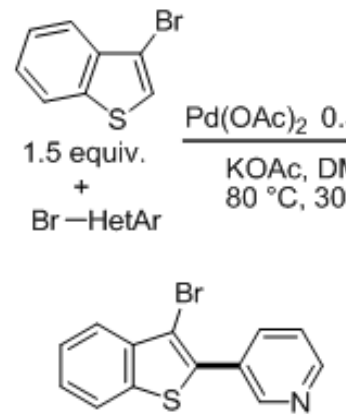

$2358 \%$

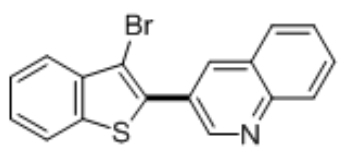

$2560 \%$ *<smiles>O=[R6]c1sc2ccccc2c1Br</smiles><smiles>Brc1c(-c2cncnc2)sc2ccccc12</smiles>

$2483 \%$ *<smiles>Brc1c(-c2cncc3ccccc23)sc2ccccc12</smiles>

$2620^{*}, 41^{\text {** }}$

* $100^{\circ} \mathrm{C}$

** $120^{\circ} \mathrm{C}, 1 \mathrm{~h}$

Scheme 5. Scope of the direct 2-arylation of 3-bromobenzothiophene with heteroaryl bromides 
In the presence of electron-rich aryl bromides such as 4-bromotoluene, disappointing results had been obtained due to complete decomposition of the reagents (see scheme 2). As the oxidative addition of aryl iodides to palladium generally requires lower reaction temperatures than aryl bromides; the reactivity of 4-iodoanisole was examined. At $80{ }^{\circ} \mathrm{C}$, this aryl iodide nicely reacts with 3-bromobenzothiophene to afford the desired product 27 in $66 \%$ yield (Scheme 6).

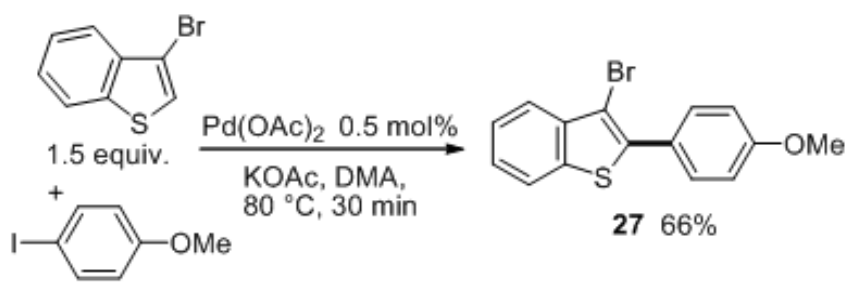

Scheme 6. Scope of the direct 2-arylation of 3-bromobenzothiophene

Then, in order to demonstrate the synthetic potential of the 2-aryl-3-bromobenzothiophenes, we investigated the reactivity of $\mathbf{7}$ and $\mathbf{1 3}$ in Pd-catalysed direct arylations using pyrrole and furan derivatives as the coupling partners (Scheme 7). The expected compounds 28 and 29 were obtained in $76 \%$ and $81 \%$ yields using $1 \mathrm{~mol} \% \mathrm{Pd}(\mathrm{OAc})_{2}$ catalyst at $130{ }^{\circ} \mathrm{C}$. No significant influence of the aryl substituents of the 2-aryl-3-bromobenzothiophenes was observed.

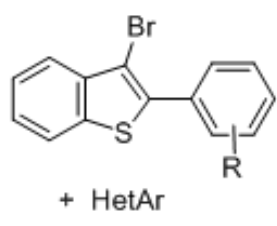

1.5 equiv.<smiles>CC(=O)c1ccc(-c2c(-c3ccc([N+](=O)[O-])cc3)sc3ccccc23)n1C</smiles>

$2876 \%$
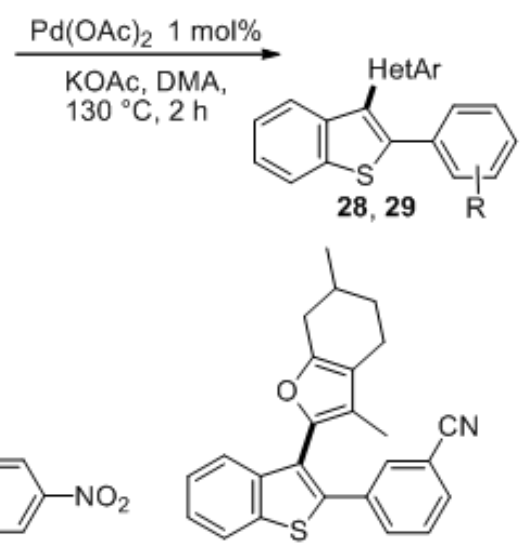

$2981 \%$

Scheme 7. Pd-catalysed direct arylations of 2-aryl-3-bromobenzothiophenes

In order to further demonstrate the potential of 2-aryl-3-bromobenzothiophenes, a Suzuki coupling reaction ${ }^{6,7}$ between 4-(3-bromobenzo[b]thiophen-2-yl)benzonitrile 1 and benzeneboronic acid was performed. At $130{ }^{\circ} \mathrm{C}$, the reaction proceeds nicely to afford $\mathbf{3 0}$ in $85 \%$ yield.

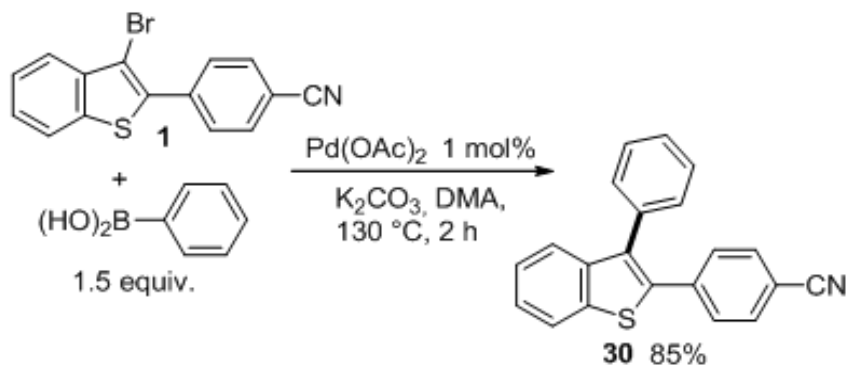

Scheme 8. Suzuki coupling with a 2-aryl-3-bromobenzothiophene 


\section{Conclusion}

In summary, we have demonstrated that using appropriate reaction conditions, a variety of aryl halides can be successfully coupled with 3-bromobenzothiophene via a palladium catalysed direct arylation without cleavage of the benzothienyl $\mathrm{C}-\mathrm{Br}$ bond. Using as little as $0.5 \mathrm{~mol} \%$ of $\mathrm{Pd}(\mathrm{OAc})_{2}$ as the catalyst precursor at $80-130{ }^{\circ} \mathrm{C}$, the direct 2-arylation of 3-bromobenzothiophene generally proceeds in high yields with aryl bromides that possess electron withdrawing groups such as cyano, nitro, propionyl, ester, acetyl, formyl, benzoyl or trifluoromethyl. Satisfactory results were also obtained using heteroaryl bromides. With 3-bromobenzothiophene, moderate reaction temperatures are mandatory to avoid unwanted oligomerization processes. This procedure which employs a quite low loading of an air-stable commercially available catalyst and an inexpensive base is economically and environmentally attractive. The major by-products are $\mathrm{AcOH} / \mathrm{KBr}$ instead of metallic salts with more classical cross-coupling procedures such as Suzuki, Stille or Negishi reactions. Moreover, no preparation of an organometallic derivative is required, reducing the number of steps and consequently the amount of waste to prepare these compounds.

\section{Experimental Section}

General Remarks: All reactions were run under argon in Schlenk tubes using vacuum lines. DMA analytical grade was not distilled before use. Potassium acetate (99+) were used. Commercial aryl bromides and 3bromobenzothiophene were used without purification. ${ }^{1} \mathrm{H}$ and ${ }^{13} \mathrm{C}$ spectrum were recorded with Bruker $400 \mathrm{MHz}$ spectrometer in $\mathrm{CDCl}_{3}$ solutions. Chemical shift are reported in ppm relative to $\mathrm{CDCl}_{3}\left(7.25\right.$ for ${ }^{1} \mathrm{H} \mathrm{NMR}$ and 77.0 for ${ }^{13} \mathrm{C}$ NMR). Flash chromatography were performed on silica gel (230-400 mesh).

\section{General procedure}

As a typical experiment, the reaction of the aryl bromide $(1 \mathrm{mmol}), 3$-bromobenzothiophene $(0.320 \mathrm{~g}, 1.5 \mathrm{mmol})$ and KOAc $(0.196 \mathrm{~g}, 2 \mathrm{mmol})$ at $80-130^{\circ} \mathrm{C}$ (see tables or schemes) during $0.5-2 \mathrm{~h}$ (see tables or schemes) in DMA $(4 \mathrm{~mL})$ in the presence of $\mathrm{Pd}(\mathrm{OAc})_{2}$ (see tables or schemes) under argon affords the corresponding product after evaporation of the solvent and filtration on silica gel (pentane:ether 1:2 except for 7, 12 and 23-26 1:10).

\section{4-(3-Bromobenzo[b]thiophen-2-yl)benzonitrile (1) $)^{15}$}

4-Bromobenzonitrile $(0.182 \mathrm{~g}, 1 \mathrm{mmol})$, 3-bromobenzothiophene $(0.320 \mathrm{~g}, 1.5 \mathrm{mmol})$ and KOAc $(0.196 \mathrm{~g}, 2$ mmol) with $\mathrm{Pd}(\mathrm{OAc})_{2}(2.24 \mathrm{mg}, 0.01 \mathrm{mmol})$ in DMA at $80{ }^{\circ} \mathrm{C}$ during $0.5 \mathrm{~h}$ affords the product 1 in $84 \%(0.264 \mathrm{~g})$ isolated yield as a white solid (mp: $92-94{ }^{\circ} \mathrm{C}$ ).

${ }^{1} \mathrm{H}$ NMR $\left(400 \mathrm{MHz}, \mathrm{CDCl}_{3}\right): \delta$ 7.93-7.87 (m, 3H), $7.85(\mathrm{~d}, J=8.2 \mathrm{~Hz}, 1 \mathrm{H}), 7.77(\mathrm{~d}, J=8.2 \mathrm{~Hz}, 2 \mathrm{H}), 7.52(\mathrm{t}, J=8.0$ $\mathrm{Hz}, 1 \mathrm{H}), 7.46(\mathrm{t}, J=8.0 \mathrm{~Hz}, 1 \mathrm{H})$.

\section{4-(3-Bromobenzothiophen-2-yl)benzaldehyde (2)}

4-Bromobenzaldehyde $(0.185 \mathrm{~g}, 1 \mathrm{mmol})$, 3-bromobenzothiophene $(0.320 \mathrm{~g}, 1.5 \mathrm{mmol})$ and KOAc $(0.196 \mathrm{~g}, 2$ mmol) with $\mathrm{Pd}(\mathrm{OAc})_{2}(2.24 \mathrm{mg}, 0.01 \mathrm{mmol})$ in DMA at $80{ }^{\circ} \mathrm{C}$ during $0.5 \mathrm{~h}$ affords the product 2 in $88 \%(0.279 \mathrm{~g})$ isolated yield as a white solid (mp: $118-122^{\circ} \mathrm{C}$ ).

${ }^{1} \mathrm{H}$ NMR $\left(400 \mathrm{MHz}, \mathrm{CDCl}_{3}\right): \delta 10.08(\mathrm{~s}, 1 \mathrm{H}), 7.98(\mathrm{~d}, J=8.2 \mathrm{~Hz}, 2 \mathrm{H}), 7.95(\mathrm{~d}, J=8.2 \mathrm{~Hz}, 2 \mathrm{H}), 7.94-7.87(\mathrm{~m}, 1 \mathrm{H})$, $7.83(\mathrm{~d}, J=8.2 \mathrm{~Hz}, 1 \mathrm{H}), 7.51(\mathrm{t}, J=8.0 \mathrm{~Hz}, 1 \mathrm{H}), 7.45(\mathrm{t}, J=8.0 \mathrm{~Hz}, 1 \mathrm{H})$.

${ }^{13} \mathrm{C} \mathrm{NMR}\left(100 \mathrm{MHz}, \mathrm{CDCl}_{3}\right): \delta$ 191.6, 139.1, 137.9, 136.5, 135.9, 130.2, 129.8, 126.1, 125.5, 124.0, 122.2, 106.4.

elemental analysis: calcd (\%) for $\mathrm{C}_{15} \mathrm{H}_{9} \mathrm{BrOS}$ (317.20): C 56.80, H 2.86; found: C 56.98, H 2.87.

\section{1-(4-(3-Bromobenzothiophen-2-yl)phenyl)ethanone (3)}

4-Bromoacetophenone (0.199 g, $1 \mathrm{mmol})$, 3-bromobenzothiophene $(0.320 \mathrm{~g}, 1.5 \mathrm{mmol})$ and KOAc $(0.196 \mathrm{~g}, 2$ mmol) with $\mathrm{Pd}(\mathrm{OAc})_{2}(2.24 \mathrm{mg}, 0.01 \mathrm{mmol})$ in DMA at $80^{\circ} \mathrm{C}$ during $0.5 \mathrm{~h}$ affords the product $3 \mathrm{in} 79 \%(0.261 \mathrm{~g})$ isolated yield as a white solid (mp: $97-100^{\circ} \mathrm{C}$ ).

${ }^{1} \mathrm{H}$ NMR (400 MHz, $\left.\mathrm{CDCl}_{3}\right): \delta 8.07(\mathrm{~d}, J=8.2 \mathrm{~Hz}, 2 \mathrm{H}), 7.96-7.80(\mathrm{~m}, 4 \mathrm{H}), 7.49(\mathrm{t}, J=8.0 \mathrm{~Hz}, 1 \mathrm{H}), 7.45(\mathrm{t}, J=8.0$ $\mathrm{Hz}, 1 \mathrm{H}), 2.66$ (s, 3H).

${ }^{13} \mathrm{C}$ NMR $\left(100 \mathrm{MHz}, \mathrm{CDCl}_{3}\right): \delta$ 197.4, 139.1, 137.8, 137.7, 136.8, 136.7, 129.8, 128.5, 126.0, 125.5, 123.9, 122.2, 106.1, 26.7.

elemental analysis: calcd (\%) for $\mathrm{C}_{16} \mathrm{H}_{11} \mathrm{BrOS}$ (331.23): C 58.02, H 3.35; found: C 58.22, H 3.47. 


\section{1-(4-(3-Bromobenzo[b]thiophen-2-yl)phenyl)propan-1-one (4)}

4-Bromopropiophenone (0.213 g, $1 \mathrm{mmol})$, 3-bromobenzothiophene $(0.320 \mathrm{~g}, 1.5 \mathrm{mmol})$ and KOAc $(0.196 \mathrm{~g}, 2$ $\mathrm{mmol})$ with $\mathrm{Pd}(\mathrm{OAc})_{2}(2.24 \mathrm{mg}, 0.01 \mathrm{mmol})$ in DMA at $80{ }^{\circ} \mathrm{C}$ during $0.5 \mathrm{~h}$ affords the product 4 in $76 \%(0.262 \mathrm{~g})$ isolated yield as a white solid (mp: $130-133^{\circ} \mathrm{C}$ ).

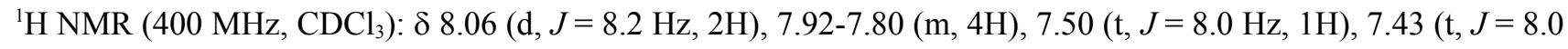
$\mathrm{Hz}, 1 \mathrm{H}), 3.06(\mathrm{t}, J=7.6 \mathrm{~Hz}, 2 \mathrm{H}), 1.27(\mathrm{q}, J=7.6 \mathrm{~Hz}, 3 \mathrm{H})$.

${ }^{13} \mathrm{C}$ NMR $\left(100 \mathrm{MHz}, \mathrm{CDCl}_{3}\right): \delta 200.1,139.1,137.8,137.5,136.9,136.6,129.8,128.2,125.9,125.4,123.9,122.2$, 106.0, 31.9, 8.2.

elemental analysis: calcd (\%) for $\mathrm{C}_{17} \mathrm{H}_{13} \mathrm{BrOS}$ (345.25): C 59.14, H 3.80; found: C 59.34, H 4.04.

\section{(4-(3-Bromobenzothiophen-2-yl)phenyl)(phenyl)methanone (5)}

4-Bromobenzophenone $(0.261 \mathrm{~g}, 1 \mathrm{mmol})$, 3-bromobenzothiophene $(0.320 \mathrm{~g}, 1.5 \mathrm{mmol})$ and KOAc $(0.196 \mathrm{~g}, 2$ $\mathrm{mmol})$ with $\mathrm{Pd}(\mathrm{OAc})_{2}(2.24 \mathrm{mg}, 0.01 \mathrm{mmol})$ in DMA at $120{ }^{\circ} \mathrm{C}$ during $1 \mathrm{~h}$ affords the product 5 in $78 \%(0.306 \mathrm{~g})$ isolated yield as a yellow solid (mp: $\left.111-114^{\circ} \mathrm{C}\right)$.

${ }^{1} \mathrm{H}$ NMR (400 MHz, $\left.\mathrm{CDCl}_{3}\right): \delta$ 7.95-7.80 (m, 8H), 7.63 (t, $\left.J=8.0 \mathrm{~Hz}, 1 \mathrm{H}\right), 7.55-7.46(\mathrm{~m}, 3 \mathrm{H}), 7.43(\mathrm{t}, J=8.0 \mathrm{~Hz}$, $1 \mathrm{H})$.

${ }^{13} \mathrm{C}$ NMR $\left(100 \mathrm{MHz}, \mathrm{CDCl}_{3}\right): \delta 196.0,139.1,137.8,137.5,137.4,137.1,136.9,132.6,130.3,130.0,129.5,128.4$, $125.9,125.5,123.9,122.2,106.1$.

elemental analysis: calcd (\%) for $\mathrm{C}_{21} \mathrm{H}_{13} \mathrm{BrOS}$ (393.30): C 64.13, H 3.33; found: C 64.29, H 3.27.

\section{Methyl 4-(3-bromobenzo[b]thiophen-2-yl)benzoate (6)}

Methyl 4-bromobenzoate $(0.215 \mathrm{~g}, 1 \mathrm{mmol})$, 3-bromobenzothiophene $(0.320 \mathrm{~g}, 1.5 \mathrm{mmol})$ and KOAc $(0.196 \mathrm{~g}, 2$ $\mathrm{mmol})$ with $\mathrm{Pd}(\mathrm{OAc})_{2}(2.24 \mathrm{mg}, 0.01 \mathrm{mmol})$ in DMA at $80^{\circ} \mathrm{C}$ during $0.5 \mathrm{~h}$ affords the product 6 in $82 \%(0.284 \mathrm{~g})$ isolated yield as a yellow solid (mp: $76-80^{\circ} \mathrm{C}$ ).

${ }^{1} \mathrm{H}$ NMR $\left(400 \mathrm{MHz}, \mathrm{CDCl}_{3}\right): \delta 8.14(\mathrm{~d}, J=8.2 \mathrm{~Hz}, 2 \mathrm{H}), 7.93-7.80(\mathrm{~m}, 4 \mathrm{H}), 7.48(\mathrm{t}, J=8.0 \mathrm{~Hz}, 1 \mathrm{H}), 7.45(\mathrm{t}, J=8.0$ $\mathrm{Hz}, 1 \mathrm{H}), 3.96(\mathrm{~s}, 3 \mathrm{H})$.

${ }^{13} \mathrm{C}$ NMR $\left(100 \mathrm{MHz}, \mathrm{CDCl}_{3}\right): \delta 166.6,139.1,137.8,137.6,136.9,130.1,129.8,129.6,125.9,125.4,123.9,122.2$, 106.0, 52.3.

elemental analysis: calcd (\%) for $\mathrm{C}_{16} \mathrm{H}_{11} \mathrm{BrO}_{2} \mathrm{~S}$ (347.23): C 55.34, H 3.19; found: C 55.20, H 3.28.

\section{3-Bromo-2-(4-nitrophenyl)benzothiophene (7)}

4-Bromonitrobenzene $(0.202 \mathrm{~g}, 1 \mathrm{mmol}), 3$-bromobenzothiophene $(0.320 \mathrm{~g}, 1.5 \mathrm{mmol})$ and KOAc $(0.196 \mathrm{~g}, 2$ $\mathrm{mmol})$ with $\mathrm{Pd}(\mathrm{OAc})_{2}(2.24 \mathrm{mg}, 0.01 \mathrm{mmol})$ in DMA at $80{ }^{\circ} \mathrm{C}$ during $0.5 \mathrm{~h}$ affords the product $7 \mathrm{in} 87 \%(0.291 \mathrm{~g})$ isolated yield as a yellow solid (mp: $150-153^{\circ} \mathrm{C}$ ).

${ }^{1} \mathrm{H}$ NMR (400 MHz, $\left.\mathrm{CDCl}_{3}\right): \delta 8.32(\mathrm{~d}, J=8.2 \mathrm{~Hz}, 2 \mathrm{H}), 7.94(\mathrm{~d}, J=8.2 \mathrm{~Hz}, 2 \mathrm{H}), 7.91(\mathrm{~d}, J=8.2 \mathrm{~Hz}, 1 \mathrm{H}), 7.84$ (d, $J=8.2 \mathrm{~Hz}, 1 \mathrm{H}), 7.55-7.35(\mathrm{~m}, 2 \mathrm{H})$.

${ }^{13} \mathrm{C}$ NMR $\left(100 \mathrm{MHz}, \mathrm{CDCl}_{3}\right): \delta 147.6,139.6,139.0,137.9,135.3,130.4,126.4,125.7,124.2,123.8,122.3,107.1$.

elemental analysis: calcd (\%) for $\mathrm{C}_{14} \mathrm{H}_{8} \mathrm{BrNO}_{2} \mathrm{~S}$ (334.19): C 50.32, H 2.41; found: C 50.17, H 2.50.

\section{3-Bromo-2-(4-chlorophenyl)benzothiophene (8)}

4-Bromochlorobenzene (0.192 g, $1 \mathrm{mmol})$, 3-bromobenzothiophene $(0.320 \mathrm{~g}, 1.5 \mathrm{mmol})$ and KOAc $(0.196 \mathrm{~g}, 2$ mmol) with $\mathrm{Pd}(\mathrm{OAc})_{2}(2.24 \mathrm{mg}, 0.01 \mathrm{mmol})$ in DMA at $130{ }^{\circ} \mathrm{C}$ during $0.5 \mathrm{~h}$ affords the product 8 in $22 \%(0.071 \mathrm{~g})$ isolated yield as a white solid (mp: $155-158^{\circ} \mathrm{C}$ ).

${ }^{1} \mathrm{H}$ NMR $\left(400 \mathrm{MHz}, \mathrm{CDCl}_{3}\right): \delta 7.87(\mathrm{~d}, J=8.2 \mathrm{~Hz}, 1 \mathrm{H}), 7.82(\mathrm{~d}, J=8.2 \mathrm{~Hz}, 1 \mathrm{H}), 7.70(\mathrm{~d}, J=8.2 \mathrm{~Hz}, 2 \mathrm{H}), 7.55-$ $7.35(\mathrm{~m}, 4 \mathrm{H})$.

${ }^{13} \mathrm{C} \mathrm{NMR}\left(100 \mathrm{MHz}, \mathrm{CDCl}_{3}\right): \delta$ 139.0, 137.6, 136.8, 134.9, 131.5, 130.9, 128.9, 125.7, 125.4, 123.8, 122.2, 105.4.

elemental analysis: calcd (\%) for $\mathrm{C}_{14} \mathrm{H}_{8} \mathrm{BrClS}$ (323.64): C 51.96, H 2.49; found: C 51.78, H 2.60.

\section{3-Bromo-2-(4-fluorophenyl)benzo[b]thiophene (9)}

4-Bromofluorobenzene $(0.175 \mathrm{~g}, 1 \mathrm{mmol}), 3$-bromobenzothiophene $(0.320 \mathrm{~g}, 1.5 \mathrm{mmol})$ and KOAc $(0.196 \mathrm{~g}, 2$ mmol) with $\mathrm{Pd}(\mathrm{OAc})_{2}(2.24 \mathrm{mg}, 0.01 \mathrm{mmol})$ in DMA at $80{ }^{\circ} \mathrm{C}$ during $0.5 \mathrm{~h}$ affords the product 9 in $8 \%(0.025 \mathrm{~g})$ 
isolated yield as a colorless oil.

${ }^{1} \mathrm{H}$ NMR $\left(400 \mathrm{MHz}, \mathrm{CDCl}_{3}\right): \delta 7.87(\mathrm{~d}, J=8.2 \mathrm{~Hz}, 1 \mathrm{H}), 7.81(\mathrm{~d}, J=8.2 \mathrm{~Hz}, 1 \mathrm{H}), 7.73(\mathrm{dd}, J=8.2,5.8 \mathrm{~Hz}, 2 \mathrm{H})$, $7.48(\mathrm{t}, J=8.0 \mathrm{~Hz}, 1 \mathrm{H}), 7.42(\mathrm{t}, J=8.0 \mathrm{~Hz}, 1 \mathrm{H}), 7.18(\mathrm{t}, J=8.2 \mathrm{~Hz}, 2 \mathrm{H})$.

\section{3-Bromo-2-(3-nitrophenyl)benzothiophene (12)}

3-Bromonitrobenzene $(0.202 \mathrm{~g}, 1 \mathrm{mmol})$, 3-bromobenzothiophene $(0.320 \mathrm{~g}, 1.5 \mathrm{mmol})$ and KOAc $(0.196 \mathrm{~g}, 2$ mmol) with $\mathrm{Pd}(\mathrm{OAc})_{2}(2.24 \mathrm{mg}, 0.01 \mathrm{mmol})$ in DMA at $80{ }^{\circ} \mathrm{C}$ during $2 \mathrm{~h}$ affords the product 12 in $83 \%(0.277 \mathrm{~g})$ isolated yield as a yellow solid (mp: $100-103{ }^{\circ} \mathrm{C}$ ).

${ }^{1} \mathrm{H}$ NMR $\left(400 \mathrm{MHz}, \mathrm{CDCl}_{3}\right): \delta 8.65(\mathrm{~s}, 1 \mathrm{H}), 8.29(\mathrm{~d}, J=8.2 \mathrm{~Hz}, 1 \mathrm{H}), 8.10(\mathrm{~d}, J=8.2 \mathrm{~Hz}, 1 \mathrm{H}), 7.91(\mathrm{~d}, J=8.2 \mathrm{~Hz}$, $1 \mathrm{H}), 7.86(\mathrm{~d}, J=8.2 \mathrm{~Hz}, 1 \mathrm{H}), 7.68(\mathrm{t}, J=8.0 \mathrm{~Hz}, 1 \mathrm{H}), 7.51(\mathrm{t}, J=8.0 \mathrm{~Hz}, 1 \mathrm{H}), 7.45(\mathrm{t}, J=8.0 \mathrm{~Hz}, 1 \mathrm{H})$.

${ }^{13} \mathrm{C}$ NMR $\left(100 \mathrm{MHz}, \mathrm{CDCl}_{3}\right): \delta 148.4,138.8,137.8,135.5,135.1,134.8,129.6,126.3,125.7,124.5,124.1,123.4$, 122.3, 106.8.

elemental analysis: calcd (\%) for $\mathrm{C}_{14} \mathrm{H}_{8} \mathrm{BrNO}_{2} \mathrm{~S}$ (334.19): C 50.32, H 2.41; found: C 50.08, H 2.24.

\section{3-(3-Bromobenzothiophen-2-yl)benzonitrile (13) ${ }^{7 \mathrm{~d}}$}

3-Bromobenzonitrile $(0.182 \mathrm{~g}, 1 \mathrm{mmol}), 3$-bromobenzothiophene $(0.320 \mathrm{~g}, 1.5 \mathrm{mmol})$ and KOAc $(0.196 \mathrm{~g}, 2$ $\mathrm{mmol})$ with $\mathrm{Pd}(\mathrm{OAc})_{2}(2.24 \mathrm{mg}, 0.01 \mathrm{mmol})$ in DMA at $80{ }^{\circ} \mathrm{C}$ during $0.5 \mathrm{~h}$ affords the product $\mathbf{1 3}$ in $69 \%(0.217 \mathrm{~g})$ isolated yield as a pink solid (mp: $98-102^{\circ} \mathrm{C}$ ).

${ }^{1} \mathrm{H}$ NMR $\left(400 \mathrm{MHz}, \mathrm{CDCl}_{3}\right): \delta 8.06(\mathrm{~s}, 1 \mathrm{H}), 7.99(\mathrm{~d}, J=8.2 \mathrm{~Hz}, 1 \mathrm{H}), 7.90(\mathrm{~d}, J=8.2 \mathrm{~Hz}, 1 \mathrm{H}), 7.84(\mathrm{~d}, J=8.2 \mathrm{~Hz}$, $1 \mathrm{H}), 7.71(\mathrm{~d}, J=8.2 \mathrm{~Hz}, 1 \mathrm{H}), 7.60(\mathrm{t}, J=8.0 \mathrm{~Hz}, 1 \mathrm{H}), 7.52(\mathrm{t}, J=8.0 \mathrm{~Hz}, 1 \mathrm{H}), 7.46(\mathrm{t}, J=8.0 \mathrm{~Hz}, 1 \mathrm{H})$.

\section{1-(3-(3-Bromobenzothiophen-2-yl)phenyl)ethanone (14)}

3-Bromoacetophenone $(0.199 \mathrm{~g}, 1 \mathrm{mmol}), 3$-bromobenzothiophene $(0.320 \mathrm{~g}, 1.5 \mathrm{mmol})$ and KOAc $(0.196 \mathrm{~g}, 2$ $\mathrm{mmol})$ with $\mathrm{Pd}(\mathrm{OAc})_{2}(2.24 \mathrm{mg}, 0.01 \mathrm{mmol})$ in DMA at $80{ }^{\circ} \mathrm{C}$ during $0.5 \mathrm{~h}$ affords the product 14 in $67 \%(0.222 \mathrm{~g})$ isolated yield as a pink solid (mp: $118-122^{\circ} \mathrm{C}$ ).

${ }^{1} \mathrm{H}$ NMR $\left(400 \mathrm{MHz}, \mathrm{CDCl}_{3}\right): \delta 8.35(\mathrm{~s}, 1 \mathrm{H}), 8.02(\mathrm{~d}, J=8.2 \mathrm{~Hz}, 1 \mathrm{H}), 7.96(\mathrm{~d}, J=8.2 \mathrm{~Hz}, 1 \mathrm{H}), 7.89(\mathrm{~d}, J=8.2 \mathrm{~Hz}$, $1 \mathrm{H}), 7.84(\mathrm{~d}, J=8.2 \mathrm{~Hz}, 1 \mathrm{H}), 7.59(\mathrm{t}, J=8.0 \mathrm{~Hz}, 1 \mathrm{H}), 7.51(\mathrm{t}, J=8.0 \mathrm{~Hz}, 1 \mathrm{H}), 7.45(\mathrm{t}, J=8.0 \mathrm{~Hz}, 1 \mathrm{H}), 2.67(\mathrm{~s}$, $3 \mathrm{H})$.

${ }^{13} \mathrm{C}$ NMR $\left(100 \mathrm{MHz}, \mathrm{CDCl}_{3}\right): \delta 197.5,139.0,137.7,137.4,136.9,134.0,133.6,129.6,129.0,128.4,125.8,125.4$, $123.8,122.2,105.8,26.7$.

elemental analysis: calcd (\%) for $\mathrm{C}_{16} \mathrm{H}_{11} \mathrm{BrOS}$ (331.23): C 58.02, H 3.35; found: C 58.31, H 3.01.

\section{3-(3-Bromobenzo[b]thiophen-2-yl)benzaldehyde (15)}

3-Bromobenzaldehyde $(0.185 \mathrm{~g}, 1 \mathrm{mmol}), 3$-bromobenzothiophene $(0.320 \mathrm{~g}, 1.5 \mathrm{mmol})$ and KOAc $(0.196 \mathrm{~g}, 2$ mmol) with $\mathrm{Pd}(\mathrm{OAc})_{2}(2.24 \mathrm{mg}, 0.01 \mathrm{mmol})$ in DMA at $80{ }^{\circ} \mathrm{C}$ during $0.5 \mathrm{~h}$ affords the product 15 in $67 \%(0.212 \mathrm{~g})$ isolated yield as a white solid (mp: $99-102{ }^{\circ} \mathrm{C}$ ).

${ }^{1} \mathrm{H}$ NMR $\left(400 \mathrm{MHz}, \mathrm{CDCl}_{3}\right): \delta 10.11(\mathrm{~s}, 1 \mathrm{H}), 8.26(\mathrm{~s}, 1 \mathrm{H}), 8.04(\mathrm{~d}, J=8.2 \mathrm{~Hz}, 1 \mathrm{H}), 7.95(\mathrm{~d}, J=8.2 \mathrm{~Hz}, 1 \mathrm{H}), 7.90$ $(\mathrm{d}, J=8.2 \mathrm{~Hz}, 1 \mathrm{H}), 7.84(\mathrm{~d}, J=8.2 \mathrm{~Hz}, 1 \mathrm{H}), 7.67(\mathrm{t}, J=8.0 \mathrm{~Hz}, 1 \mathrm{H}), 7.51(\mathrm{t}, J=8.0 \mathrm{~Hz}, 1 \mathrm{H}), 7.45(\mathrm{t}, J=8.0 \mathrm{~Hz}$, $1 \mathrm{H})$.

${ }^{13} \mathrm{C}$ NMR $\left(100 \mathrm{MHz}, \mathrm{CDCl}_{3}\right): \delta 191.6,138.9,137.7,136.7,136.5,135.3,134.2,131.0,129.6,129.4,125.9,125.5$, 123.9, 122.3, 106.0.

elemental analysis: calcd (\%) for $\mathrm{C}_{15} \mathrm{H}_{9} \mathrm{BrOS}$ (317.20): C 56.80, H 2.86; found: C 56.74, H 2.64.

\section{2-(3,5-Bis(trifluoromethyl)phenyl)-3-bromobenzothiophene (16)}

From 3,5-bis(trifluoromethyl)bromobenzene $(0.293 \mathrm{~g}, 1 \mathrm{mmol}), 3$-bromobenzothiophene $(0.320 \mathrm{~g}, 1.5 \mathrm{mmol})$ and KOAc $(0.196 \mathrm{~g}, 2 \mathrm{mmol})$ with $\mathrm{Pd}(\mathrm{OAc})_{2}(2.24 \mathrm{mg}, 0.01 \mathrm{mmol})$ in DMA at $80{ }^{\circ} \mathrm{C}$ during $0.5 \mathrm{~h}$ affords the product 16 in $80 \%(0.340 \mathrm{~g})$ isolated yield as a colorless oil.

${ }^{1} \mathrm{H}$ NMR (400 MHz, $\left.\mathrm{CDCl}_{3}\right): \delta 8.22(\mathrm{~s}, 2 \mathrm{H}), 7.93(\mathrm{~s}, 1 \mathrm{H}), 7.92(\mathrm{~d}, J=8.2 \mathrm{~Hz}, 1 \mathrm{H}), 7.85(\mathrm{~d}, J=8.2 \mathrm{~Hz}, 1 \mathrm{H}), 7.55(\mathrm{t}$, $J=8.0 \mathrm{~Hz}, 1 \mathrm{H}), 7.48(\mathrm{t}, J=8.0 \mathrm{~Hz}, 1 \mathrm{H})$.

${ }^{13} \mathrm{C}$ NMR $\left(100 \mathrm{MHz}, \mathrm{CDCl}_{3}\right): \delta 138.8,137.8,135.3,134.4,132.3$ (q, $\left.J=33.0 \mathrm{~Hz}\right), 129.7,126.5,125.8,124.3$, $123.1(\mathrm{q}, J=272.6 \mathrm{~Hz}), 122.3,122.2,107.3$.

elemental analysis: calcd (\%) for $\mathrm{C}_{16} \mathrm{H}_{7} \mathrm{BrF}_{6} \mathrm{~S}$ (425.19): C 45.20, H 1.66; found: C 45.00, H 1.51. 


\section{3-Bromo-2-(4-nitro-3-(trifluoromethyl)phenyl)benzothiophene (17)}

4-Bromo-1-nitro-2-(trifluoromethyl)benzene $(0.270 \mathrm{~g}, 1 \mathrm{mmol}), 3$-bromobenzothiophene $(0.320 \mathrm{~g}, 1.5 \mathrm{mmol})$ and $\mathrm{KOAc}(0.196 \mathrm{~g}, 2 \mathrm{mmol})$ with $\mathrm{Pd}(\mathrm{OAc})_{2}(2.24 \mathrm{mg}, 0.01 \mathrm{mmol})$ in DMA at $80{ }^{\circ} \mathrm{C}$ during $0.5 \mathrm{~h}$ affords the product $\mathbf{1 7}$ in $83 \%(0.333 \mathrm{~g})$ isolated yield as a yellow solid (mp: $\left.97-99^{\circ} \mathrm{C}\right)$.

${ }^{1} \mathrm{H}$ NMR $\left(400 \mathrm{MHz}, \mathrm{CDCl}_{3}\right): \delta 8.25(\mathrm{~s}, 1 \mathrm{H}), 8.12(\mathrm{~d}, J=8.2 \mathrm{~Hz}, 1 \mathrm{H}), 8.02(\mathrm{~d}, J=8.2 \mathrm{~Hz}, 1 \mathrm{H}), 7.93(\mathrm{~d}, J=8.2 \mathrm{~Hz}$, $1 \mathrm{H}), 7.87(\mathrm{~d}, J=8.2 \mathrm{~Hz}, 1 \mathrm{H}), 7.55(\mathrm{t}, J=8.0 \mathrm{~Hz}, 1 \mathrm{H}), 7.50(\mathrm{t}, J=8.0 \mathrm{~Hz}, 1 \mathrm{H})$.

${ }^{13} \mathrm{C}$ NMR $\left(100 \mathrm{MHz}, \mathrm{CDCl}_{3}\right): \delta 147.4,138.9,138.3,138.0,133.8,133.7,129.0$ (q, $\left.J=5.4 \mathrm{~Hz}\right), 126.9,126.0,125.7$, $124.5,124.4$ (q, $J=34.2 \mathrm{~Hz}), 122.5,121.6(\mathrm{q}, J=273.9 \mathrm{~Hz}), 108.1$.

elemental analysis: calcd (\%) for $\mathrm{C}_{15} \mathrm{H}_{7} \mathrm{BrF}_{3} \mathrm{NO}_{2} \mathrm{~S}$ (402.19): C 44.80, H 1.75; found: C 45.07, $\mathrm{H} 1.81$.

\section{3-Bromo-2-(3-fluorophenyl)benzothiophene (18)}

3-Bromofluorobenzene $(0.175 \mathrm{~g}, 1 \mathrm{mmol})$, 3-bromobenzothiophene $(0.320 \mathrm{~g}, 1.5 \mathrm{mmol})$ and KOAc $(0.196 \mathrm{~g}, 2$ mmol) with $\mathrm{Pd}(\mathrm{OAc})_{2}(2.24 \mathrm{mg}, 0.01 \mathrm{mmol})$ in DMA at $80{ }^{\circ} \mathrm{C}$ during $0.5 \mathrm{~h}$ affords the product 18 in $12 \%(0.037 \mathrm{~g})$ isolated yield as a colorless oil.

${ }^{1} \mathrm{H}$ NMR (400 MHz, $\left.\mathrm{CDCl}_{3}\right): \delta 7.89(\mathrm{~d}, J=8.2 \mathrm{~Hz}, 1 \mathrm{H}), 7.83(\mathrm{~d}, J=8.2 \mathrm{~Hz}, 1 \mathrm{H}), 7.55-7.38(\mathrm{~m}, 5 \mathrm{H}), 7.13(\mathrm{t}, J=7.8$ $\mathrm{Hz}, 1 \mathrm{H})$.

${ }^{13} \mathrm{C}$ NMR $\left(100 \mathrm{MHz}, \mathrm{CDCl}_{3}\right): \delta 162.2(\mathrm{~d}, J=247.0 \mathrm{~Hz}), 158.0,139.1,137.7,130.1(\mathrm{~d}, J=8.3 \mathrm{~Hz}), 125.8,125.4$ (m), 123.8, 122.2, $116.6(\mathrm{~d}, J=23.0 \mathrm{~Hz}), 115.7(\mathrm{~d}, J=21.2 \mathrm{~Hz}), 105.6$.

elemental analysis: calcd (\%) for $\mathrm{C}_{14} \mathrm{H}_{8} \mathrm{BrFS}$ (307.18): C 54.74, H 2.63; found: C 54.70, H 2.89.

\section{2-(3-Bromobenzo[b]thiophen-2-yl)benzaldehyde (19)}

2-Bromobenzaldehyde $(0.185 \mathrm{~g}, 1 \mathrm{mmol})$, 3-bromobenzothiophene $(0.320 \mathrm{~g}, 1.5 \mathrm{mmol})$ and KOAc $(0.196 \mathrm{~g}, 2$ $\mathrm{mmol})$ with $\mathrm{Pd}(\mathrm{OAc})_{2}(2.24 \mathrm{mg}, 0.01 \mathrm{mmol})$ in DMA at $80^{\circ} \mathrm{C}$ during $0.5 \mathrm{~h}$ affords the product $19 \mathrm{in} 80 \%(0.254 \mathrm{~g})$ isolated yield as a yellow oil.

${ }^{1} \mathrm{H}$ NMR (400 MHz, CDCl $): \delta 10.01(\mathrm{~s}, 1 \mathrm{H}), 8.11(\mathrm{~d}, J=8.2 \mathrm{~Hz}, 1 \mathrm{H}), 7.88(\mathrm{~d}, J=8.2 \mathrm{~Hz}, 1 \mathrm{H}), 7.86(\mathrm{~d}, J=8.2 \mathrm{~Hz}$, $1 \mathrm{H}), 7.70(\mathrm{t}, J=8.0 \mathrm{~Hz}, 1 \mathrm{H}), 7.62(\mathrm{t}, J=8.0 \mathrm{~Hz}, 1 \mathrm{H}), 7.57-7.52(\mathrm{~m}, 2 \mathrm{H}), 7.45(\mathrm{t}, J=8.0 \mathrm{~Hz}, 1 \mathrm{H})$.

${ }^{13} \mathrm{C}$ NMR $\left(100 \mathrm{MHz}, \mathrm{CDCl}_{3}\right): \delta 190.9,138.5,138.0,136.1,134.5,133.9,133.6,132.0,129.7,127.8,126.1,125.7$, $123.7,122.2,109.8$.

elemental analysis: calcd (\%) for $\mathrm{C}_{15} \mathrm{H}_{9} \mathrm{BrOS}$ (317.20): C 56.80, H 2.86; found: C 56.89, H 2.94.

\section{2-(3-Bromobenzo[b]thiophen-2-yl)benzonitrile (20) ${ }^{4}$}

2-Bromobenzonitrile $(0.182 \mathrm{~g}, 1 \mathrm{mmol}), 3$-bromobenzothiophene $(0.320 \mathrm{~g}, 1.5 \mathrm{mmol})$ and KOAc $(0.196 \mathrm{~g}, 2$ mmol) with $\mathrm{Pd}(\mathrm{OAc})_{2}(2.24 \mathrm{mg}, 0.01 \mathrm{mmol})$ in DMA at $80{ }^{\circ} \mathrm{C}$ during $2 \mathrm{~h}$ affords the product 20 in $88 \%(0.276 \mathrm{~g})$ isolated yield as a white solid (mp: $161-163^{\circ} \mathrm{C}$ ).

${ }^{1} \mathrm{H}$ NMR $\left(400 \mathrm{MHz}, \mathrm{CDCl}_{3}\right): \delta 7.91(\mathrm{~d}, J=8.2 \mathrm{~Hz}, 1 \mathrm{H}), 7.85(\mathrm{~d}, J=8.2 \mathrm{~Hz}, 1 \mathrm{H}), 7.83(\mathrm{~d}, J=8.2 \mathrm{~Hz}, 1 \mathrm{H}), 7.71(\mathrm{t}$, $J=8.0 \mathrm{~Hz}, 1 \mathrm{H}), 7.63(\mathrm{~d}, J=8.2 \mathrm{~Hz}, 1 \mathrm{H}), 7.57(\mathrm{t}, J=8.0 \mathrm{~Hz}, 1 \mathrm{H}), 7.54(\mathrm{t}, J=8.0 \mathrm{~Hz}, 1 \mathrm{H}), 7.45(\mathrm{t}, J=8.0 \mathrm{~Hz}, 1 \mathrm{H})$.

\section{3-Bromo-2-(naphthalen-1-yl)benzo[b]thiophene (21)}

1-Bromonaphthalene (0.207, $1 \mathrm{mmol})$, 3-bromobenzothiophene $(0.320 \mathrm{~g}, 1.5 \mathrm{mmol})$ and KOAc $(0.196 \mathrm{~g}, 2 \mathrm{mmol})$ with $\mathrm{Pd}(\mathrm{OAc})_{2}(2.24 \mathrm{mg}, 0.01 \mathrm{mmol})$ in DMA at $120^{\circ} \mathrm{C}$ during $1 \mathrm{~h}$ affords the product 21 in $61 \%(0.207 \mathrm{~g})$ isolated yield as a yellow oil.

${ }^{1} \mathrm{H}$ NMR (400 MHz, $\left.\mathrm{CDCl}_{3}\right): \delta 7.98(\mathrm{~d}, J=8.2 \mathrm{~Hz}, 1 \mathrm{H}), 7.94(\mathrm{~d}, J=8.2 \mathrm{~Hz}, 1 \mathrm{H}), 7.92(\mathrm{~d}, J=8.2 \mathrm{~Hz}, 1 \mathrm{H}), 7.88(\mathrm{~d}$, $J=8.2 \mathrm{~Hz}, 1 \mathrm{H}), 7.80(\mathrm{~d}, J=8.2 \mathrm{~Hz}, 1 \mathrm{H}), 7.64-7.43(\mathrm{~m}, 6 \mathrm{H})$.

${ }^{13} \mathrm{C}$ NMR $\left(100 \mathrm{MHz}, \mathrm{CDCl}_{3}\right): \delta 138.7,138.3,136.9,133.5,131.8,130.5,129.7,129.3,128.4,126.7,126.2,126.0$, $125.5,125.3,125.1,123.5,122.2,108.5$.

elemental analysis: calcd (\%) for $\mathrm{C}_{18} \mathrm{H}_{11} \mathrm{BrS}$ (339.25): C 63.73, H 3.27; found: C 63.80, H 3.51.

\section{2-(Anthracen-9-yl)-3-bromobenzothiophene (22)}

9-Bromoanthracene $(0.257,1 \mathrm{mmol})$, 3-bromobenzothiophene $(0.320 \mathrm{~g}, 1.5 \mathrm{mmol})$ and KOAc $(0.196 \mathrm{~g}, 2 \mathrm{mmol})$ with $\mathrm{Pd}(\mathrm{OAc})_{2}(2.24 \mathrm{mg}, 0.01 \mathrm{mmol})$ in DMA at $80{ }^{\circ} \mathrm{C}$ during $0.5 \mathrm{~h}$ affords the product 22 in $64 \%(0.249 \mathrm{~g})$ isolated yield as a yellow solid (mp: $105-108^{\circ} \mathrm{C}$ ).

${ }^{1} \mathrm{H}$ NMR $\left(400 \mathrm{MHz}, \mathrm{CDCl}_{3}\right): \delta 8.52(\mathrm{~d}, J=8.2 \mathrm{~Hz}, 1 \mathrm{H}), 8.46(\mathrm{~s}, 1 \mathrm{H}), 8.01(\mathrm{~d}, J=8.2 \mathrm{~Hz}, 1 \mathrm{H}), 7.92(\mathrm{~d}, J=8.2 \mathrm{~Hz}$, $2 \mathrm{H}), 7.85(\mathrm{~d}, J=8.2 \mathrm{~Hz}, 2 \mathrm{H}), 7.61(\mathrm{t}, J=7.7 \mathrm{~Hz}, 1 \mathrm{H}), 7.55-7.45(\mathrm{~m}, 5 \mathrm{H})$. 
${ }^{13} \mathrm{C}$ NMR $\left(100 \mathrm{MHz}, \mathrm{CDCl}_{3}\right): \delta 139.1,138.1,132.2,131.7,130.6,129.4,128.6,127.6,127.2,126.3,125.6,125.4$, $124.0,122.3,122.2,110.8$.

elemental analysis: calcd (\%) for $\mathrm{C}_{22} \mathrm{H}_{13} \mathrm{BrS}$ (389.31): C 67.87, H 3.37; found: C 67.99, H 3.50.

\section{3-(3-Bromobenzo[b]thiophen-2-yl)pyridine (23)}

3-Bromopyridine $(0.158 \mathrm{~g}, 1 \mathrm{mmol}), 3$-bromobenzothiophene $(0.320 \mathrm{~g}, 1.5 \mathrm{mmol})$ and KOAc $(0.196 \mathrm{~g}, 2 \mathrm{mmol})$ with $\mathrm{Pd}(\mathrm{OAc})_{2}(2.24 \mathrm{mg}, 0.01 \mathrm{mmol})$ in DMA at $80{ }^{\circ} \mathrm{C}$ during $0.5 \mathrm{~h}$ affords the product 23 in $58 \%(0.168 \mathrm{~g})$ isolated yield as a yellow solid (mp: $119-122^{\circ} \mathrm{C}$ ).

${ }^{1} \mathrm{H}$ NMR (400 MHz, $\left.\mathrm{CDCl}_{3}\right): \delta 9.03(\mathrm{bs}, 1 \mathrm{H}), 8.69(\mathrm{bs}, 1 \mathrm{H}), 8.10(\mathrm{~d}, J=8.0 \mathrm{~Hz}, 1 \mathrm{H}), 7.90(\mathrm{~d}, J=7.8 \mathrm{~Hz}, 1 \mathrm{H}), 7.85$ $(\mathrm{d}, J=7.9 \mathrm{~Hz}, 1 \mathrm{H}), 7.51(\mathrm{t}, J=7.7 \mathrm{~Hz}, 1 \mathrm{H}), 7.50-7.43(\mathrm{~m}, 2 \mathrm{H})$.

${ }^{13} \mathrm{C}$ NMR $\left(100 \mathrm{MHz}, \mathrm{CDCl}_{3}\right): \delta 150.0,149.6,139.0,138.0,137.1,134.3,126.1,125.7,124.0,122.4,106.7$.

elemental analysis: calcd (\%) for $\mathrm{C}_{13} \mathrm{H}_{8} \mathrm{BrSN}$ (290.18): C 53.81, H 2.78; found: C 53.70, H 2.99.

\section{5-(3-Bromobenzothiophen-2-yl)pyrimidine (24)}

5-Bromopyrimidine (0.159 g, $1 \mathrm{mmol}), 3$-bromobenzothiophene $(0.320 \mathrm{~g}, 1.5 \mathrm{mmol})$ and KOAc $(0.196 \mathrm{~g}, 2 \mathrm{mmol})$ with $\mathrm{Pd}(\mathrm{OAc})_{2}(2.24 \mathrm{mg}, 0.01 \mathrm{mmol})$ in DMA at $100{ }^{\circ} \mathrm{C}$ during $0.5 \mathrm{~h}$ affords the product 24 in $83 \%(0.241 \mathrm{~g})$ isolated yield as a white solid (mp: $136-140{ }^{\circ} \mathrm{C}$ ).

${ }^{1} \mathrm{H}$ NMR $\left(400 \mathrm{MHz}, \mathrm{CDCl}_{3}\right): \delta 9.27(\mathrm{~s}, 1 \mathrm{H}), 9.15(\mathrm{~s}, 2 \mathrm{H}), 7.91(\mathrm{~d}, J=8.2 \mathrm{~Hz}, 1 \mathrm{H}), 7.87(\mathrm{~d}, J=8.2 \mathrm{~Hz}, 1 \mathrm{H}), 7.54$ (t, $J=8.0 \mathrm{~Hz}, 1 \mathrm{H}), 7.49(\mathrm{t}, J=8.0 \mathrm{~Hz}, 1 \mathrm{H})$.

${ }^{13} \mathrm{C}$ NMR $\left(100 \mathrm{MHz}, \mathrm{CDCl}_{3}\right): \delta 158.1,156.7,138.7,138.1,130.4,127.5,126.5,125.8,124.2,122.4,107.8$.

elemental analysis: calcd (\%) for $\mathrm{C}_{12} \mathrm{H}_{7} \mathrm{BrN}_{2} \mathrm{~S}$ (291.17): C 49.50, H 2.42; found: C 49.75, H 2.51.

\section{3-(3-Bromobenzothiophen-2-yl)quinoline (25)}

3-Bromoquinoline $(0.208 \mathrm{~g}, 1 \mathrm{mmol}), 3$-bromobenzothiophene $(0.320 \mathrm{~g}, 1.5 \mathrm{mmol})$ and KOAc $(0.196 \mathrm{~g}, 2 \mathrm{mmol})$ with $\mathrm{Pd}(\mathrm{OAc})_{2}(2.24 \mathrm{mg}, 0.01 \mathrm{mmol})$ in DMA at $100{ }^{\circ} \mathrm{C}$ during $0.5 \mathrm{~h}$ affords the product 25 in $60 \%(0.204 \mathrm{~g})$ isolated yield as a pink solid (mp: $\left.81-84{ }^{\circ} \mathrm{C}\right)$.

${ }^{1} \mathrm{H}$ NMR $\left(400 \mathrm{MHz}, \mathrm{CDCl}_{3}\right): \delta 9.30(\mathrm{~s}, 1 \mathrm{H}), 8.53(\mathrm{~d}, J=1.8 \mathrm{~Hz}, 1 \mathrm{H}), 8.18(\mathrm{~d}, J=8.5 \mathrm{~Hz}, 1 \mathrm{H}), 7.93(\mathrm{~d}, J=8.5 \mathrm{~Hz}$, $2 \mathrm{H}), 7.87(\mathrm{~d}, J=8.5 \mathrm{~Hz}, 1 \mathrm{H}), 7.80(\mathrm{t}, J=8.0 \mathrm{~Hz}, 1 \mathrm{H}), 7.63(\mathrm{t}, J=8.0 \mathrm{~Hz}, 1 \mathrm{H}), 7.54(\mathrm{t}, J=8.0 \mathrm{~Hz}, 1 \mathrm{H}), 7.46(\mathrm{t}, J=$ $8.0 \mathrm{~Hz}, 1 \mathrm{H})$.

${ }^{13} \mathrm{C}$ NMR $\left(100 \mathrm{MHz}, \mathrm{CDCl}_{3}\right): \delta 150.2,147.2,138.9,138.0,136.8,134.4,130.5,129.2,128.2,127.5,126.6,126.0$, $125.6,123.9,122.3,106.8$.

elemental analysis: calcd (\%) for $\mathrm{C}_{17} \mathrm{H}_{10} \mathrm{BrNS}$ (340.24): C 60.01, H 2.96; found: C 60.24, H 3.09.

\section{4-(3-Bromobenzo[b]thiophen-2-yl)isoquinoline (26)}

4-Bromoisoquinoline (0.208 g, $1 \mathrm{mmol})$, 3-bromobenzothiophene $(0.320 \mathrm{~g}, 1.5 \mathrm{mmol})$ and KOAc $(0.196 \mathrm{~g}, 2$ mmol) with $\mathrm{Pd}(\mathrm{OAc})_{2}(2.24 \mathrm{mg}, 0.01 \mathrm{mmol})$ in DMA at $120^{\circ} \mathrm{C}$ during $1 \mathrm{~h}$ affords the product 26 in $41 \%(0.139 \mathrm{~g})$ isolated yield as a yellow solid (mp: $90-93{ }^{\circ} \mathrm{C}$ ).

${ }^{1} \mathrm{H}$ NMR $\left(400 \mathrm{MHz}, \mathrm{CDCl}_{3}\right): \delta 9.40(\mathrm{~s}, 1 \mathrm{H}), 8.65(\mathrm{~s}, 1 \mathrm{H}), 8.14(\mathrm{~d}, J=8.5 \mathrm{~Hz}, 1 \mathrm{H}), 7.94(\mathrm{~d}, J=8.5 \mathrm{~Hz}, 1 \mathrm{H}), 7.90(\mathrm{~d}$, $J=8.5 \mathrm{~Hz}, 1 \mathrm{H}), 7.86(\mathrm{~d}, J=8.5 \mathrm{~Hz}, 1 \mathrm{H}), 7.79(\mathrm{t}, J=8.0 \mathrm{~Hz}, 1 \mathrm{H}), 7.73(\mathrm{t}, J=8.0 \mathrm{~Hz}, 1 \mathrm{H}), 7.55(\mathrm{t}, J=8.0 \mathrm{~Hz}, 1 \mathrm{H})$, $7.50(\mathrm{t}, J=8.0 \mathrm{~Hz}, 1 \mathrm{H})$.

${ }^{13} \mathrm{C}$ NMR $\left(100 \mathrm{MHz}, \mathrm{CDCl}_{3}\right): \delta 152.5,143.2,138.8,138.2,134.9,132.4,132.0,128.4,128.2,126.1,125.6,125.2$, 123.7, 122.3, 109.9.

elemental analysis: calcd (\%) for $\mathrm{C}_{17} \mathrm{H}_{10} \mathrm{BrNS}$ (340.24): C 60.01, H 2.96; found: $\mathrm{C} 60.17$, H 3.11.

\section{3-Bromo-2-(4-methoxyphenyl)benzo[b]thiophene $(27)^{15}$}

4-Iodoanisole $(0.234 \mathrm{~g}, 1 \mathrm{mmol}), 3$-bromobenzothiophene $(0.320 \mathrm{~g}, 1.5 \mathrm{mmol})$ and KOAc $(0.196 \mathrm{~g}, 2 \mathrm{mmol})$ with $\mathrm{Pd}(\mathrm{OAc})_{2}(2.24 \mathrm{mg}, 0.01 \mathrm{mmol})$ in DMA at $80{ }^{\circ} \mathrm{C}$ during $0.5 \mathrm{~h}$ affords the product 27 in $66 \%(0.211 \mathrm{~g})$ isolated yield as a yellow solid (mp: $78-82^{\circ} \mathrm{C}$ ).

${ }^{1} \mathrm{H}$ NMR $\left(400 \mathrm{MHz}, \mathrm{CDCl}_{3}\right): \delta 7.85(\mathrm{~d}, J=8.2 \mathrm{~Hz}, 1 \mathrm{H}), 7.80(\mathrm{~d}, J=8.2 \mathrm{~Hz}, 1 \mathrm{H}), 7.71(\mathrm{~d}, J=8.2 \mathrm{~Hz}, 2 \mathrm{H}), 7.47(\mathrm{t}$, $J=7.7 \mathrm{~Hz}, 1 \mathrm{H}), 7.40(\mathrm{t}, J=7.7 \mathrm{~Hz}, 1 \mathrm{H}), 7.01(\mathrm{~d}, J=8.2 \mathrm{~Hz}, 2 \mathrm{H}), 3.88(\mathrm{~s}, 3 \mathrm{H})$. 
3-Bromo-2-(4-nitrophenyl)benzothiophene $7(0.334 \mathrm{~g}, 1 \mathrm{mmol})$, methyl 1-methylpyrrole-2-carboxylate $(0.208 \mathrm{~g}$, $1.5 \mathrm{mmol})$ and $\mathrm{KOAc}(0.196 \mathrm{~g}, 2 \mathrm{mmol})$ at $130^{\circ}$ during $2 \mathrm{~h}$ in DMA $(4 \mathrm{~mL})$ in the presence of $\mathrm{Pd}(\mathrm{OAc})_{2}(2.24 \mathrm{mg}$, $0.01 \mathrm{mmol}$ ) under argon affords the corresponding product $\mathbf{2 8}$ after evaporation of the solvent and filtration on silica gel (pentane:ether 1:10) in $76 \%(0.298 \mathrm{~g})$ yield as a yellow solid (mp: $\left.72-75^{\circ} \mathrm{C}\right)$.

${ }^{1} \mathrm{H}$ NMR $\left(400 \mathrm{MHz}, \mathrm{CDCl}_{3}\right): \delta 8.16(\mathrm{~d}, J=8.2 \mathrm{~Hz}, 2 \mathrm{H}), 7.91(\mathrm{~d}, J=8.2 \mathrm{~Hz}, 1 \mathrm{H}), 7.55(\mathrm{~d}, J=8.2 \mathrm{~Hz}, 1 \mathrm{H}), 7.50-$ $7.35(\mathrm{~m}, 4 \mathrm{H}), 7.12(\mathrm{~d}, J=4.0 \mathrm{~Hz}, 1 \mathrm{H}), 6.27(\mathrm{~d}, J=4.0 \mathrm{~Hz}, 1 \mathrm{H}), 3.86(\mathrm{~s}, 3 \mathrm{H}), 3.48(\mathrm{~s}, 3 \mathrm{H})$.

${ }^{13} \mathrm{C}$ NMR $\left(100 \mathrm{MHz}, \mathrm{CDCl}_{3}\right): \delta 161.7,141.6,139.1,130.9,130.1,129.7,128.9,125.4,124.8,123.9,123.8,123.7$, $122.2,118.5,117.2,111.6,51.2,34.0$.

elemental analysis: calcd (\%) for $\mathrm{C}_{21} \mathrm{H}_{16} \mathrm{~N}_{2} \mathrm{O}_{4} \mathrm{~S}$ (392.43): C 64.27, H 4.11; found: C 64.30, H 4.12.

\section{3-(3-(3,6-Dimethyl-4,5,6,7-tetrahydrobenzofuran-2-yl)benzo[b]thiophen-2-yl)benzonitrile (29)}

3-(3-Bromobenzothiophen-2-yl)benzonitrile $13(0.314 \mathrm{~g}, 1 \mathrm{mmol})$, menthofuran $(0.225 \mathrm{~g}, 1.5 \mathrm{mmol})$ and KOAc $(0.196 \mathrm{~g}, 2 \mathrm{mmol})$ at $130{ }^{\circ} \mathrm{C}$ during $2 \mathrm{~h}$ in DMA $(4 \mathrm{~mL})$ in the presence of $\mathrm{Pd}(\mathrm{OAc})_{2}(2.24 \mathrm{mg}, 0.01 \mathrm{mmol})$ under argon affords the corresponding product 29 after evaporation of the solvent and filtration on silica gel (pentane:ether 1:2) in $81 \%(0.310 \mathrm{~g})$ yield as a white solid (mp: $\left.156-159{ }^{\circ} \mathrm{C}\right)$.

${ }^{1} \mathrm{H}$ NMR (400 MHz, $\left.\mathrm{CDCl}_{3}\right): \delta$ 7.90-7.82 (m, 1H), 7.78-7.73 (m, 1H), 7.65-7.60 (m, 2H), 7.56 (d, $\left.J=8.0 \mathrm{~Hz}, 1 \mathrm{H}\right)$, 7.48-7.36 (m, 3H), 2.75-2.65 (m, 1H), 2.40-2.10 (m, 3H), $2.17(\mathrm{~s}, 3 \mathrm{H}), 2.00-1.70(\mathrm{~m}, 2 \mathrm{H}), 1.50-1.20(\mathrm{~m}, 1 \mathrm{H}), 1.12$ $(\mathrm{d}, J=7.5 \mathrm{~Hz}, 3 \mathrm{H})$.

${ }^{13} \mathrm{C}$ NMR $\left(100 \mathrm{MHz}, \mathrm{CDCl}_{3}\right): \delta 151.0,140.9,140.2,138.5,138.4,136.2,132.9,132.1,131.0,129.3,125.2,124.9$, $124.3,122.1,119.0,118.9,118.5,112.7,31.5,31.2,29.6,21.5,20.1,8.8$.

elemental analysis: calcd (\%) for $\mathrm{C}_{25} \mathrm{H}_{21} \mathrm{NOS}$ (383.51): C 78.30, H 5.52; found: C 78.25, H 5.68.

\section{4-(3-Phenylbenzo[b]thiophen-2-yl)benzonitrile (30)}

The reaction of 4-(3-bromobenzo[b]thiophen-2-yl)benzonitrile $1(0.314 \mathrm{~g}, 1 \mathrm{mmol})$, phenylboronic acid $(0.183 \mathrm{~g}$, $1.5 \mathrm{mmol})$ and $\mathrm{K}_{2} \mathrm{CO}_{3}(0.276 \mathrm{~g}, 2 \mathrm{mmol})$ at $130{ }^{\circ} \mathrm{C}$ during $2 \mathrm{~h}$ in DMA $(4 \mathrm{~mL})$ in the presence of $\mathrm{Pd}(\mathrm{OAc})_{2}(2.2$ $\mathrm{mg}, 0.01 \mathrm{mmol}$ ) under argon affords the corresponding product $\mathbf{3 0}$ after evaporation of the solvent and filtration on silica gel (pentane:ether 1:2) in 85\% (0.264 g) isolated yield as a white solid (mp: 200-204 ${ }^{\circ} \mathrm{C}$ ).

${ }^{1} \mathrm{H}$ NMR (400 MHz, $\left.\mathrm{CDCl}_{3}\right): \delta 7.90(\mathrm{~d}, J=8.2 \mathrm{~Hz}, 1 \mathrm{H}), 7.60(\mathrm{~d}, J=8.2 \mathrm{~Hz}, 1 \mathrm{H}), 7.52(\mathrm{~d}, J=8.2 \mathrm{~Hz}, 2 \mathrm{H}), 7.48-$ $7.28(\mathrm{~m}, 9 \mathrm{H})$.

${ }^{13} \mathrm{C}$ NMR $\left(100 \mathrm{MHz}, \mathrm{CDCl}_{3}\right): \delta 140.6,139.1,139.0,136.9,135.3,134.8,132.1,130.2,130.0,129.0,128.0,125.4$, $124.8,123.8,122.2,118.6,111.1$.

elemental analysis: calcd (\%) for $\mathrm{C}_{21} \mathrm{H}_{13} \mathrm{NS}$ (311.40): C 81.00, H 4.21; found: C 80.69, H 4.01.

Supporting Information for this article is available online at http://www.thiemeconnect.com/products/ejournals/journal/10.1055/s-00000084.

\section{Acknowledgment}

We thank the Centre National de la Recherche Scientifique, "Rennes Metropole" and "UTIQUE" for providing financial support.

\section{References}

(a) Brzozowski, A. M.; Pike, A. C. W.; Dauter, Z.; Hubbard, R. E.; Bonn, T.; Engstrom, O.; Ohman, L.; Greene, G. L.; Gustafsson, J.-

- $\quad$ A.; Carlquist M. Nature 1997, 389, 753-758; (b) Overk, C. R.; Peng, K.-W.; Asghodom, R. T.; Kastrati, I.; Lantvit, D. D.; Qin, Z.; Frasor, J.; Bolton, J. L.; Thatcher G. R. J. ChemMedChem 2007, 2, 1520-1526.

(a) Ohta, A.; Akita, Y.; Ohkuwa, T.; Chiba, M.; Fukunaga, R.; Miyafuji, A.; Nakata, T.; Tani, N.; Aoyagi, Y. Heterocycles 1990, 31, 1951-1958; (b) Aoyagi, Y.; Inoue, A.; Koizumi, I.; Hashimoto, R.; Tokunaga, K.; Gohma, K.; Komatsu, J.; Sekine, K.; Miyafuji, A.; Kunoh, J.; Honma, R.; Akita, Y.; Ohta, A. Heterocycles 1992, 33, 257-272; (c) Alberico, D.; Scott, M. E.; Lautens, M. Chem. Rev. 2007, 107, 174-238; (d) Satoh, T.; Miura, M. Chem. Lett. 2007, 36, 200-205; (e) Campeau, L.-C.; Stuart, D. R.; Fagnou, K. Aldrichimica Acta 2007, 40, 35-41; (f) Seregin, I. V.; Gevorgyan, V. Chem. Soc. Rev. 2007, 36, 1173-1193; (g) Li, B.-J.; Yang, S.-D.; Shi, Z.-J. Synlett 2008, 949-957; (h) Lewis, C.; Bergman, R. G.; Ellman, J. A. Acc. Chem. Res. 2008, 41, 1013-1025; (i) Bellina, F.; Rossi, R. Tetrahedron 2009, 65, 10269-10310; (j) Ackermann, L.; Vicente, R.; Kapdi, A. Angew. Chem. Int. Ed. 2009, 48, 9792-9826; (k) Chen, X.; Engle, K. M.; Wang, D.-H.; Yu, J.-Q. Angew. Chem. Int. Ed. 2009, 48, 5094-5115; (1) McGlacken, G. P.; Bateman, L. M. Chem. Soc. Rev. 2009, 38, 2447-2464; (m) Joucla, L.; Djakovitch, L. Adv. Synth. Catal. 2009, 351, 673-714; (n) Sun, C.-L.; Li, B.J.; Shi, Z.-J. Chem. Commun. 2010, 46, 677-685; (o) Ackermann, L. Chem. Rev. 2011, 111, 1315-1345; (p) McMurray, L.; O’Hara, F.; Gaunt, M. J. Chem. Soc. Rev. 2011, 40, 1885-1898; (q) Kuhl, N.; Hopkinson, M. N.; Wencel-Delord, J.; Glorius, F. Angew. Chem. Int. Ed. 2012, 51, 10236-10254; (r) Yamaguchi, J.; Yamaguchi, A. D.; Itami, K. Angew. Chem., Int. Ed. 2012, 51, 8960-9009; (s)

Mousseau, J. J.; Charette, A. B. Acc. Chem. Res. 2012, 46, 412-424 t) Neufeldt, S. R.; Sanford, M. S. Acc. Chem. Res. 2012, 45, 936946; (u) Wencel-Delord, J.; Glorius, F. Nature Chem. 2013, 5, 369-375; (v) Rossi, R.; Bellina, F.; Lessi, M.; Manzini, C. Adv. Synth. 
Catal. 2014, 356, 17-117; (w) He, M.; Soulé, J. F.; Doucet, H. ChemCatChem 2014, 6, 1824-1859; (x) Zhang, M.; Zhang, Y.; Jie, X.; Zhao, H.; Li, G.; Su, W. Org. Chem. Front. 2014, 1, 843-895; (y) Yuan, K.; Soulé, J. F.; Doucet, H. ACS Catal. 2015, 5, $978-991$.

- For selected examples of palladium-catalysed intermolecular direct arylations of benzothiophenes: (a) Pivsa-Art, S.; Satoh, T.; Kawamura, Y.; Miura, M.; Nomura, M. Bull. Chem. Soc. Jpn. 1998, 71, 467-473; (b) Fournier Dit Chabert, J.; Joucla, L.; David, E.; Lemaire, M. Tetrahedron 2004, 60, 3221-3230; (c) David, E.; Perrin, J.; Pellet-Rostaing, S.; Fournier dit Chabert, J.; Lemaire, M. J. Org. Chem. 2005, 70, 3569-3573; (d) Nakano, M.; Satoh, T.; Miura, M. J. Org. Chem. 2006, 71, 8309-8311; (e) Chiong, H. A.; Daugulis, O. Org. Lett. 2007, 9, 1449-1451; (f) Watanabe, H.; Kumagai, J.; Tsurugi, H.; Satoh, T.; Miura, M. Chem. Lett. 2007, 36, 1336-1337; (g) Liegault, B.; Lapointe, D.; Caron, L.; Vlassova, A.; Fagnou, K. J. Org. Chem. 2009, 74, 1826-1834; (h) Tamba, S.; Okubo, Y.; Tanaka, S.; Monguchi, D.; Mori, A. J. Org. Chem. 2010, 75, 6998-7001; (i) Lapointe, D.; Markiewicz, T.; Whipp, C. J.; Toderian, A.; Fagnou, K. J. Org. Chem. 2011, 76, 749-759; (j) Baghbanzadeh, M.; Pilger, C.; Kappe, C. O. J. Org. Chem. 2011, 76, 8138-8142; (k) Takeda, D.; Yamashita, M.; Hirano, K.; Satoh, T.; Miura, M. Chem. Lett. 2011, 40, 1015-1017; (1) Hu, P.; Zhang, M.; Jie, X.; Su, W. Angew. Chem., Int. Ed. 2012, 51, 227-231; (m) Ghosh, D.; Lee, H. M. Org. Lett. 2012, 14, 5534-5537; (n) Dao-Huy, T.; Haider, M.; Glatz, F.; Schnurch, M.; Mihovilovic, M. D. Eur. J. Org. Chem. 2014, 8119-8125.

- Zhao, L.; Bruneau, C.; Doucet, H. Tetrahedron 2013, 69, 7082-7089.

- $\quad$ Palkowitz, A. D.; Glasebrook, A. L.; Thrasher, K. J.; Hauser, K. L.; Short, L. L.; Phillips, D. L.; Muehl, B. S.; Sato, M.; Shetler, P. K.; Cullinan, G. J.; Pell, T. R.; Bryant, H. U. J. Med. Chem. 1997, 40, 1407-1416.

- (a) Li, J. J; Gribble, G. W. Palladium in Heterocyclic Chemistry, Pergamon: Amsterdam, 2000; (b) Modern Arylation Methods, Ed.: Ackermann, L. Wiley-VCH, Weinheim, 2009.

- $\quad$ For Suzuki couplings with 2,3-dibromobenzothiophenes: (a) Heynderickx, A.; Samat, A.; Guglielmetti, R. Synthesis 2002, 213-216; (b) Chauhan, J.; Monteil, A. R.; Patterson, S. E. Heterocycl. Commun. 2010, 16, 241-244; (c) Weymiens, W.; Zaal, M.; Slootweg, J. C.; Ehlers, A. W.; Lammertsma, K. Inorg. Chem. 2011, 50, 8516-8523; (d) Chelucci, G.; Baldino, S.; Ruiu, A. J. Org. Chem. 2012, 77 , 9921-9925; (e) Hung, T. Q.; Dang, T. T.; Villinger, A.; Sung, T. V.; Langer, P. Org. Biomol. Chem. 2012, 10, 9041-9044.

- $\quad$ For Stille couplings with 2,3-dibromobenzothiophenes: Hao, X.; Liang, M.; Cheng, X.; Pian, X.; Sun, Z.; Xue, S. Org. Lett. 2011, 13, 5424-5427.

- $\quad$ For Negishi couplings with 2,3-dihalobenzothiophenes: (a) Kienle, M.; Unsinn, A.; Knochel, P. Angew. Chem., Int. Ed. 2010, 49, 4751-4754; (b) Zhao, H.; Dankwardt, J. W.; Koenig, S. G.; Singh, S. P. Tetrahedron Lett. 2012, 53, 166-169.

- $\quad$ Mosrin, M.; Monzon, G.; Bresser, T.; Knochel, P. Chem. Commun. 2009, 5615-5617.

- $\quad$ For palladium-catalysed direct arylations with phosphine-free palladium catalyst: (a) Roger, J.; Požgan, F.; Doucet H. Green Chem. 2009, 11, 425-432; (b) Fu, H. Y.; Chen, L.; Doucet H. J. Org. Chem. 2012, 77, 4473-4478.

- $\quad$ Cantat, T.; Génin, E.; Giroud, C.; Meyer, G.; Jutand, A. J. Organomet. Chem. 2003, 687, 365-376.

- Carey, J. S.; Laffan, D.; Thomson C.; Williams, M. T. Org. Biomol. Chem. 2006, 4, 2337-2347.

- Campeau, L. C.; Fagnou, K.; Chem. Soc. Rev. 2007, 36, 1058-1068.

- $\quad$ Lu, W.-D.; Wu, M.-J. Tetrahedron 2007, 63, 356-362. 